\title{
ON THE CRITICAL ONE COMPONENT REGULARITY FOR 3-D NAVIER-STOKES SYSTEM: GENERAL CASE
}

\author{
JEAN-YVES CHEMIN, PING ZHANG, AND ZHIFEI ZHANG
}

\begin{abstract}
Let us consider an initial data $v_{0}$ for the homogeneous incompressible 3D NavierStokes equation with vorticity belonging to $L^{\frac{3}{2}} \cap L^{2}$. We prove that if the solution associated with $v_{0}$ blows up at a finite time $T^{\star}$, then for any $p$ in $] 4, \infty\left[\right.$, and any unit vector $e$ of $\mathbb{R}^{3}$, the $L^{p}$ norm in time with value in $\dot{H}^{\frac{1}{2}+\frac{2}{p}}$ of $(v \mid e)_{\mathbb{R}^{3}}$ blows up at $T^{\star}$.
\end{abstract}

Keywords: Incompressible Navier-Stokes Equations, Blow-up criteria, Anisotropic Littlewood-Paley Theory

AMS Subject Classification (2000): 35Q30, 76D03

\section{INTRODUCTION}

In this work, we investigate necessary conditions for breakdown of regularity of regular solutions to the following 3-D homogeneous incompressible Navier-Stokes system

$$
(N S) \quad\left\{\begin{array}{l}
\partial_{t} v+\operatorname{div}(v \otimes v)-\Delta v+\nabla \Pi=0, \quad(t, x) \in \mathbb{R}^{+} \times \mathbb{R}^{3}, \\
\operatorname{div} v=0, \\
\left.v\right|_{t=0}=v_{0},
\end{array}\right.
$$

where $v=\left(v^{1}, v^{2}, v^{3}\right)$ stands for the velocity of the fluid and $\Pi$ for the pressure. We shall study necessary conditions for blowing up in the framework of Fujita and Kato solutions. Let us sum up the fact about this theory introduced in [7] by H. Fujita and T. Kato that will be relevant in our work.

Theorem 1.1. Let $v_{0}$ be in the homogenneous Sobolev space $\dot{H}^{\frac{1}{2}}$. There exists a unique maximal solution $v$ in the space $C\left(\left[0, T^{*}\left[; \dot{H}^{\frac{1}{2}}\right) \cap L_{\mathrm{loc}}^{2}\left(\left[0, T^{\star}\left[; \dot{H}^{\frac{3}{2}}\right)\right.\right.\right.\right.$. If $T^{\star}$ is finite, then we have, for any $p$ in $[2, \infty[$

$$
\int_{0}^{T^{\star}}\|v(t, \cdot)\|_{\dot{H}^{\frac{1}{2}+\frac{2}{p}}}^{p} d t=\infty
$$

The limiting case when $p=\infty$ namely that fact that if there is blow up in finite time $T^{\star}$, then $\limsup _{t \rightarrow T^{\star}}\|v(t)\|_{\dot{H}^{\frac{1}{2}}}$ is infinite is a consequence of the work [6] of L. Escauriaza, G. Seregin and V. Šverák.

In all that follows, we consider initial data $v_{0}$ with vorticity $\Omega_{0} \stackrel{\text { def }}{=} \nabla \times v_{0}$ belonging to $L^{\frac{3}{2}}$. Let us mention that dual Sobolev embedding implies that $L^{\frac{3}{2}} \hookrightarrow \dot{H}^{-\frac{1}{2}}$ which together with Biot-Savart law ensures that $v_{0}$ belongs to $\dot{H}^{\frac{1}{2}}$. Let us introduce the following family of spaces.

Definition 1.1. For $r$ in $\left.] \frac{3}{2}, 2\right]$, we denote by $\mathcal{V}^{r}$ the space of divergence free vector fields with the vorticity of which belongs to $L^{\frac{3}{2}} \cap L^{r}$.

Date: $2 / 22 / 2015$. 
Let us remark that, if we denote

$$
\alpha(r) \stackrel{\text { def }}{=} \frac{1}{r}-\frac{1}{2}
$$

the dual Sobolev embedding $L^{r} \hookrightarrow \dot{H}^{-3 \alpha(r)}$ implies that the vector field $\mathcal{V}^{r}$ is included into $\dot{H}^{\frac{1}{2}} \cap \dot{H}^{1-3 \alpha(r)}$.

The purpose of this work is to generalize the following result proved by the first two authors in $[5]$.

Theorem 1.2. Let us consider an initial data $v_{0}$ in $\mathcal{V}^{\frac{3}{2}}$, let us consider the unique maximal solution $v$ associated with $v_{0}$ given by Theorem 1.1. If its lifespan $T^{\star}$ is finite, then we have, for any $p$ in $] 4,6\left[\right.$ and any unit vector $e$ in $\mathbb{R}^{3}$,

$$
\int_{0}^{T^{\star}}\left\|(v(t) \mid e)_{\mathbb{R}^{3}}\right\|_{\dot{H}^{\frac{1}{2}+\frac{2}{p}}}^{p} d t=\infty
$$

We refer to [5] for a detailed introduction about the history of the results involving such "anisotropic" norm for the description of blow up. The purpose of the present work is to drop the restriction on $p$ supposing that the initial data is more regular. Namely, we prove the following theorem.

Theorem 1.3. Let us consider an initial data $v_{0}$ in $\mathcal{V}^{2}$, let us consider the unique maximal solutio $n v$ associated with $v_{0}$ given by Theorem 1.1. If its lifespan $T^{\star}$ is finite, then we have, for any $p$ in $] 4, \infty\left[\right.$ and any unit vector $e$ in $\mathbb{R}^{3}$,

$$
\int_{0}^{T^{\star}}\left\|(v(t) \mid e)_{\mathbb{R}^{3}}\right\|_{\dot{H}^{\frac{1}{2}+\frac{2}{p}}}^{p} d t=\infty .
$$

Let us compare this theorem with the preceding one. Theorem 1.2 deals with solution the regularity of which is exactly at the scaling of $(N S)$. Theorem 1.3 deals with solutions which are continuous in time with value in $\dot{H}^{\frac{1}{2}} \cap \dot{H}^{1}$. But the blow up condition is much better. Indeed, the bigger $p$ is, the better the blow up condition is. Let us recall that in the case when we control the norm of all component, the blow up condition about $L_{t}^{p}\left(\dot{H}^{\frac{1}{2}+\frac{2}{p}}\right)$ is elementary for finite $p$. The case when $p$ is infinite, namely that fact that if there is blow up in finite time $T^{\star}$, then $\limsup _{t \rightarrow T^{\star}}\|v(t)\|_{\dot{H}^{\frac{1}{2}}}$ is infinite is a consequence of the work [6] of L. Escauriaza, G. Seregin and V. Šverák. It is a deep result the proof of which uses strongly the particular structure of the Navier-Stokes equation.

Let us mention that the method presented here seems far away from proving the limiting case when $p$ is infinite. Let us point out that we have no idea about the following problem: let us assume that for some unit vector $e$ of $\mathbb{R}^{3},\left\|\left(v_{0} \mid e\right)_{\mathbb{R}^{3}}\right\|_{H^{\frac{1}{2}}}$ is small with respect to some universal constant, does it imply that there is no blow up for the Fujita-Kato solution of $(N S)$ ?

\section{IDEAS OF THE PROOF AND STRUCTURE OF THE PAPER}

First of all, let us mention that we do not prove directly Theorem 1.3 but in fact the following one, which obviously implies Theorem 1.3.

Theorem 2.1. Let us consider $r$ in $\left[3 / 2,2\right.$ [ and an initial data $v_{0}$ in $\mathcal{V}^{r}$. If the lifespan $T^{\star}$ of the unique maximal solution $v$ of $(N S)$ given by Theorem 1.1 is finite, then we have, for 
any $p$ in $] 4, \frac{2 r}{2-r}\left[\right.$ and any unit vector $e$ in $\mathbb{R}^{3}$,

$$
\int_{0}^{T^{\star}}\left\|(v(t) \mid e)_{\mathbb{R}^{3}}\right\|_{\dot{H}^{\frac{1}{2}+\frac{2}{p}}}^{p} d t=\infty .
$$

The case when $r=\frac{3}{2}$ is exactly Theorem 1.2 .

Let us explain the strategy of the proof of Theorem 2.1. We first remark that it makes no restriction to assume that the unit vector $e$ is the vertical vector $e_{3} \stackrel{\text { def }}{=}(0,0,1)$. We follow essentially the same strategy as that in [5] up to some differences due to the fact that the regularity of the solution $v$ is higher than the one given by the scaling of the equation.

The first point consists in rewriting the homogeneous incompressible Navier-Stokes equation in terms of two unknowns:

- the third component of the vorticity $\Omega$, which we denote by

$$
\omega=\partial_{1} v^{2}-\partial_{2} v^{1}
$$

and which can be understood as the $2 \mathrm{D}$ vorticity for the vector field $v^{\mathrm{h}} \stackrel{\text { def }}{=}\left(v^{1}, v^{2}\right)$, - the quantity $\partial_{3} v^{3}$ which is $-\operatorname{div}_{h} v^{\mathrm{h}}=-\partial_{1} v^{1}-\partial_{2} v^{2}$ because $v$ is divergence free.

Immediate computations give

$$
(\widetilde{N S})\left\{\begin{array}{c}
\partial_{t} \omega+v \cdot \nabla \omega-\Delta \omega=\partial_{3} v^{3} \omega+\partial_{2} v^{3} \partial_{3} v^{1}-\partial_{1} v^{3} \partial_{3} v^{2} \\
\partial_{t} \partial_{3} v^{3}+v \cdot \nabla \partial_{3} v^{3}-\Delta \partial_{3} v^{3}+\partial_{3} v \cdot \nabla v^{3}=-\partial_{3}^{2} \Delta^{-1}\left(\sum_{\ell, m=1}^{3} \partial_{\ell} v^{m} \partial_{m} v^{\ell}\right) .
\end{array}\right.
$$

Let us analyse this formulation of the Navier-Stokes system keeping in mind that we already have control of $v^{3}$ in the norm $L_{T}^{p}\left(\dot{H}^{\frac{1}{2}+\frac{2}{p}}\right)$. Let us first introduce the notations

$$
\nabla_{\mathrm{h}}^{\perp}=\left(-\partial_{2}, \partial_{1}\right), \quad \Delta_{\mathrm{h}}=\partial_{1}^{2}+\partial_{2}^{2}, \quad v_{\text {curl }}^{\mathrm{h}} \stackrel{\text { def }}{=} \nabla_{\mathrm{h}}^{\perp} \Delta_{\mathrm{h}}^{-1} \omega \quad \text { and } \quad v_{\text {div }}^{\mathrm{h}} \stackrel{\text { def }}{=}-\nabla_{\mathrm{h}} \Delta_{\mathrm{h}}^{-1} \partial_{3} v^{3} .
$$

Then we have, using the Biot-Savart's law in the horizontal variables

$$
v^{\mathrm{h}}=v_{\text {curl }}^{\mathrm{h}}+v_{\text {div }}^{\mathrm{h}} .
$$

Let us concentrate on the equation on $\omega$. As we have no a priori control on $\omega$, quadratic terms in this equation of $(\widetilde{N S})$ seems dangerous. In fact, there is only one term of this type which is $v_{\text {curl }}^{\mathrm{h}} \cdot \nabla_{\mathrm{h}} \omega$. A way to get rid of it is to use an energy type estimate and the divergence free condition on $v$. Instead of working with scaling invariant norms as that in [5], namely performing a $L^{\frac{3}{2}}$ energy estimate for $\omega$, here we shall perform a $L^{r}$ energy estimate for $\omega$. This is based on the following lemma.

Lemma 2.1. Let $r$ be in $] 1,2\left[\right.$ and $a_{0}$ a function in $L^{r}$. Let us consider a function $f$ in $L_{\text {loc }}^{1}\left(\mathbb{R}^{+} ; L^{r}\right)$ and $v$ a divergence free vector field in $L_{\text {loc }}^{2}\left(\mathbb{R}^{+} ; L^{\infty}\right)$. If a solves

$$
\left(T_{v}\right) \quad\left\{\begin{array}{c}
\partial_{t} a-\Delta a+v \cdot \nabla a=f \\
a_{\mid t=0}=a_{0}
\end{array}\right.
$$

then $|a|^{r / 2}$ belongs to $L_{\text {loc }}^{\infty}\left(\mathbb{R}^{+} ; L^{2}\right) \cap L_{\text {loc }}^{2}\left(\mathbb{R}^{+} ; \dot{H}^{1}\right)$ and

$$
\begin{aligned}
\frac{1}{r} \int_{\mathbb{R}^{3}}|a(t, x)|^{r} d x & +(r-1) \int_{0}^{t} \int_{\mathbb{R}^{3}}\left|\nabla a\left(t^{\prime}, x\right)\right|^{2}\left|a\left(t^{\prime}, x\right)\right|^{r-2} d x d t^{\prime} \\
& =\frac{1}{r} \int_{\mathbb{R}^{3}}\left|a_{0}(x)\right|^{r} d x+\int_{0}^{t} \int_{\mathbb{R}^{3}} f\left(t^{\prime}, x\right) a\left(t^{\prime}, x\right)\left|a\left(t^{\prime}, x\right)\right|^{r-2} d x d t^{\prime} .
\end{aligned}
$$


For the proof, see for instance [5], Lemma 3.1.

The terms on the right-hand side of the equation on $\omega$ in $(\widetilde{N S})$ can be decomposed as $\mathcal{L} \omega+F$ with

$$
\mathcal{L} \omega \stackrel{\text { def }}{=} \partial_{3} v^{3} \omega+\partial_{2} v^{3} \partial_{3} v_{\text {curl }}^{1}-\partial_{1} v^{3} \partial_{3} v_{\text {curl }}^{2} \quad \text { and } \quad F \stackrel{\text { def }}{=} \partial_{2} v^{3} \partial_{3} v_{\text {div }}^{1}-\partial_{1} v^{3} \partial_{3} v_{\text {div }}^{2}
$$

These two terms are different. The term $\mathcal{L} \omega$ is linear with respect to $\omega$ and thus can be estimated with quantities related to scaling invariant space after some Gronwall lemma. The term $F$ is a forcing term. It is quadratic with respect to $v_{3}$ and will be estimated with one term related to scaling 0 and another term related to the scaling corresponding to the vorticity in $L^{r}$.

It remains to examine the second equation of $(\widetilde{N S})$, which is

$$
\partial_{t} \partial_{3} v^{3}+v \cdot \nabla \partial_{3} v^{3}-\Delta \partial_{3} v^{3}+\partial_{3} v \cdot \nabla v^{3}=-\partial_{3}^{2} \Delta^{-1}\left(\sum_{\ell, m=1}^{3} \partial_{\ell} v^{m} \partial_{m} v^{\ell}\right) .
$$

The main feature of this equation is that it contains only one quadratic term with respect to $\omega$, namely the term

$$
-\partial_{3}^{2} \Delta^{-1}\left(\sum_{\ell, m=1}^{2} \partial_{\ell} v_{\text {curl }}^{m} \partial_{m} v_{\text {curl }}^{\ell}\right) .
$$

Because we control $v^{3}$ on some norm, a way to get rid of this term is to perform an energy estimate on $\partial_{3} v^{3}$, namely an estimate on

$$
\left\|\partial_{3} v^{3}(t)\right\|_{\mathcal{H}}
$$

for an adapted Hilbert space $\mathcal{H}$. Indeed, we hope that if we control $v^{3}$, we can control terms of the type

$$
\left(\partial_{3}^{2} \Delta^{-1}\left(\partial_{\ell} v_{\rho m c u r l}^{m} \partial_{m} v_{\text {curl }}^{\ell}\right) \mid \partial_{3} v^{3}\right)_{\mathcal{H}}
$$

with quadratic terms in $\omega$ and thus it fits with $\left\|\partial_{3} v^{3}\right\|_{\mathcal{H}}^{2}$ so that we can hope to close the estimate. Again here, the scaling helps us for the choice of the Hilbert space $\mathcal{H}$. The scaling of $\mathcal{H}$ must be the scaling of $\dot{H}^{-3 \alpha(r)}$ for $\alpha(r)$ given by (1.2). Moreover, because of the operator $\nabla_{\mathrm{h}} \Delta_{\mathrm{h}}^{-1}$, it is natural to measure horizontal derivatives and vertical derivatives differently. This leads to the following definition.

Definition 2.1. For $\left(s, s^{\prime}\right)$ in $\mathbb{R}^{2}, \dot{H}^{s, s^{\prime}}$ denotes the space of tempered distribution a such that

$$
\|a\|_{\dot{H}^{s, s^{\prime}}}^{2} \stackrel{\text { def }}{=} \int_{\mathbb{R}^{3}}\left|\xi_{\mathrm{h}}\right|^{2 s}\left|\xi_{3}\right|^{2 s^{\prime}}|\widehat{a}(\xi)|^{2} d \xi<\infty \quad \text { with } \quad \xi_{\mathrm{h}}=\left(\xi_{1}, \xi_{2}\right) .
$$

For $\alpha(r)$ given by $(1.2)$ and $\theta$ in $] 0, \alpha(r)\left[\right.$, we denote $\mathcal{H}^{\theta, r} \stackrel{\text { def }}{=} \dot{H}^{-3 \alpha(r)+\theta,-\theta}$.

We want to emphasize the fact that anisotropy in the regularity is highly related to the divergence free condition. Indeed, let us consider a divergence free vector field $w=\left(w^{\mathrm{h}}, w^{3}\right)$ in $\dot{H}^{1-3 \alpha(r)}$ and let us estimate $\left\|\partial_{3} w^{3}\right\|_{\mathcal{H}^{\theta, r}}$. By definition of the $\mathcal{H}^{\theta, r}$ norm, we have

$$
\left\|\partial_{3} w^{3}\right\|_{\mathcal{H}^{\theta, r}}^{2}=A_{L}+A_{H} \quad \text { with } \quad A_{L} \stackrel{\text { def }}{=} \int_{\left|\xi_{\mathrm{h}}\right| \leq\left|\xi_{3}\right|}\left|\xi_{\mathrm{h}}\right|^{-6 \alpha(r)+2 \theta}\left|\xi_{3}\right|^{-2 \theta}\left|\mathcal{F}\left(\partial_{3} w^{3}\right)(\xi)\right|^{2} d \xi .
$$

In the case when $\left|\xi_{\mathrm{h}}\right| \geq\left|\xi_{3}\right|$, since $\left.\theta \in\right] 0, \alpha(r)[$, we write that

$$
A_{H} \leq \int_{\mathbb{R}^{3}}\left|\xi_{3}\right|^{2(1-3 \alpha(r))}\left|\widehat{w}^{3}(\xi)\right|^{2} d \xi \leq\left\|w^{3}\right\|_{\dot{H}^{1-3 \alpha(r)}}^{2} .
$$


In the case when $\left|\xi_{\mathrm{h}}\right| \leq\left|\xi_{3}\right|$, we use divergence free condition and write that

$$
\begin{aligned}
A_{L} & \leq a m p ; \int_{\left|\xi_{\mathrm{h}}\right| \leq\left|\xi_{3}\right|}\left|\xi_{\mathrm{h}}\right|^{-6 \alpha(r)}\left|\mathcal{F}\left(\operatorname{div}_{\mathrm{h}} w^{\mathrm{h}}\right)(\xi)\right|^{2} d \xi \\
& \leq \int_{\mathbb{R}^{3}}\left|\xi_{\mathrm{h}}\right|^{2(1-3 \alpha(r))}\left|\widehat{w}^{\mathrm{h}}(\xi)\right|^{2} d \xi=\left\|w^{\mathrm{h}}\right\|_{\dot{H}^{1-3 \alpha(r)}}^{2} .
\end{aligned}
$$

Thus for any divergence free vector field $w$ in $\dot{H}^{1-3 \alpha(r)}$, we have

$$
\left\|\partial_{3} w^{3}\right\|_{\mathcal{H}^{\theta, r}} \leq C\|w\|_{\dot{H}^{1-3 \alpha(r)}} .
$$

To use the space efficiently in the proof, we need to rely them on anisotropic Littlewood-Paley theory and also anisotropic Besov spaces. This is the purpose of the third section.

The first step of the proof of Theorem 2.1 is the following proposition:

Proposition 2.1. Let $v_{0}$ be in $\mathcal{V}^{r}$; let us consider a solution $v$ of $(N S)$ given by Theorem 1.1. Then for any $p$ in $] 4, \frac{2 r}{2-r}[$ and any $\theta$ in $] 0, \alpha(r)\left[\right.$, a constant $C$ exists such that, for any $t<T^{\star}$,

$$
\begin{aligned}
\frac{1}{r}\left\|\omega_{\frac{r}{2}}(t)\right\|_{L^{2}}^{2}+\frac{r}{r^{2}} \int_{0}^{t}\left\|\nabla \omega_{\frac{r}{2}}\left(t^{\prime}\right)\right\|_{L^{2}}^{2} d t^{\prime} \leq\left(\frac{1}{r}\left\|\left|\omega_{0}\right|^{\frac{r}{2}}\right\|_{L^{2}}^{2}\right. \\
\left.\quad+\left(\int_{0}^{t}\left\|\partial_{3}^{2} v^{3}\left(t^{\prime}\right)\right\|_{\mathcal{H}^{\theta, r}}^{2} d t^{\prime}\right)^{\frac{r}{2}}\right) \exp \left(C \int_{0}^{t}\left\|v^{3}\left(t^{\prime}\right)\right\|_{\dot{H}^{\frac{1}{2}+\frac{2}{p}}}^{p} d t^{\prime}\right) .
\end{aligned}
$$

Here and in all that follows, for scalar function $a$ and for $\alpha$ in the interval ]0,1[, we always denote

$$
a_{\alpha} \stackrel{\text { def }}{=} \frac{a}{|a|}|a|^{\alpha} .
$$

Up to some technical difficulties, the proof of this proposition follows essentially the lines of the analogous proposition in [5]; it is the purpose of the fourth section.

Next we want to control $\left\|\partial_{3}^{2} v^{3}\right\|_{L_{t}^{2}\left(\mathcal{H}^{\theta, r}\right)}$. As already explained, a way to get rid of the only quadratic term in $\omega$, namely

$$
-\partial_{3}^{2} \Delta^{-1}\left(\sum_{\ell, m=1}^{2} \partial_{\ell} v_{\text {curl }}^{m} \partial_{m} v_{\text {curl }}^{\ell}\right)
$$

is to perform an energy estimate for the norm $\mathcal{H}^{\theta, r}$.

Proposition 2.2. Let $v_{0}$ be in $\mathcal{V}^{r}$; let us consider a solution $v$ of $(N S)$ given by Theorem 1.1. For any $p$ in $] 4, \frac{2 r}{2-r}[$ and $\theta$ in $] 3 \alpha(r)-\frac{2}{p}, \alpha(r)\left[\right.$, a constant $C$ exists such that for any $t<T^{\star}$, we have

$$
\begin{aligned}
& \left\|\partial_{3} v^{3}(t)\right\|_{\mathcal{H}^{\theta, r}}^{2}+\int_{0}^{t}\left\|\nabla \partial_{3} v^{3}\left(t^{\prime}\right)\right\|_{\mathcal{H}^{\theta, r}}^{2} d t^{\prime} \leq C \exp \left(C \int_{0}^{t}\left\|v^{3}\left(t^{\prime}\right)\right\|_{\dot{H}^{\frac{1}{2}+\frac{2}{p}}}^{p} d t^{\prime}\right) \\
& \text { pourtout uad } \quad \times\left(\left\|\Omega_{0}\right\|_{L^{r}}^{2}+\int_{0}^{t}\left(\left\|v^{3}\left(t^{\prime}\right)\right\|_{\dot{H}^{\frac{1}{2}+\frac{2}{p}}}\left\|\omega_{\frac{r}{2}}\left(t^{\prime}\right)\right\|_{L^{2}}^{2\left(2 \alpha(r)+\frac{1}{p}\right)}\left\|\nabla \omega_{\frac{r}{2}}\left(t^{\prime}\right)\right\|_{L^{2}}^{\frac{2}{p^{\prime}}}\right.\right. \\
& \left.\left.+\left\|v^{3}\left(t^{\prime}\right)\right\|_{\dot{H}^{\frac{1}{2}+\frac{2}{p}}}^{2}\left\|\omega_{\frac{r}{2}}\left(t^{\prime}\right)\right\|_{L^{2}}^{4\left(\alpha(r)+\frac{1}{p}\right)}\left\|\nabla \omega_{\frac{r}{2}}\left(t^{\prime}\right)\right\|_{L^{2}}^{2\left(1-\frac{2}{p}\right)}\right) d t^{\prime}\right) .
\end{aligned}
$$

Here and in all that follows, $p^{\prime}$ denotes the conjugate number of $p$ so that $\frac{1}{p^{\prime}}=1-\frac{1}{p}$. 
The proof of this proposition is different from the one of the analogous proposition in [5]. In the framework of that article, only laws of product of quantities related to scaling 0 were used. Here, two different scalings are involved and we do use the structure of the transport term $v \cdot \nabla$ to prove propagation estimate in a quasi-linear spirit. The term $\left(v^{\mathrm{h}} \cdot \nabla_{\mathrm{h}} \partial_{3} v^{3} \mid \partial_{3} v^{3}\right)_{\mathcal{H}^{\theta, r}}$ requires a particular care (see forthcoming Lemma 5.2). The proof of Proposition 2.2 is the purpose of the fifth section.

A non-standard Gronwall type argument allows to deduce from Propositions 2.1 and 2.2 that we control the quantities

$$
\|\omega\|_{L_{t}^{\infty}\left(L^{r}\right)}, \quad \int_{0}^{t}\left\|\nabla \omega_{\frac{r}{2}}\left(t^{\prime}\right)\right\|_{L^{2}}^{2} d t^{\prime}, \quad \int_{0}^{t}\left\|\partial_{3}^{2} v^{3}\left(t^{\prime}\right)\right\|_{\mathcal{H}^{\theta, r}}^{2} d t^{\prime} \quad \text { and } \quad \int_{0}^{t}\left\|v^{3}\left(t^{\prime}\right)\right\|_{\dot{H}^{\frac{1}{2}+\frac{2}{p}}}^{p} d t^{\prime} .
$$

Let us also point out that these quantities have different scaling; the quantity

$$
\int_{0}^{t}\left\|v^{3}\left(t^{\prime}\right)\right\|_{\dot{H}^{\frac{1}{2}+\frac{2}{p}}}^{p} d t^{\prime}
$$

is scaling invariant and the quantities

$$
\|\omega\|_{L_{t}^{\infty}\left(L^{r}\right)}, \quad \int_{0}^{t}\left\|\nabla \omega_{\frac{r}{2}}\left(t^{\prime}\right)\right\|_{L^{2}}^{2} d t^{\prime} \quad \text { and } \quad \int_{0}^{t}\left\|\partial_{3}^{2} v^{3}\left(t^{\prime}\right)\right\|_{\mathcal{H}^{\theta, r}}^{2} d t^{\prime}
$$

are the scaling of the norm $L_{t}^{\infty}\left(\dot{H}^{1-3 \alpha(r)}\right)$. Biot-Savart law in the horizontal variable allows to prove that all the above quantities in (2.11) prevents the solution $v$ of $(N S)$ from blowing up. The details of all this is the purpose of the last section.

\section{Non linear inequalities and Littlewood-Paley Analysis}

In this section, we recall or prove estimates that will be useful later on and recall the basics of anisotropic Littlewood-Paley theory. As a warm up, let us establish some Sobolev type inequalities which involve the regularities of $a_{\frac{r}{2}}$ and $\nabla a_{\frac{r}{2}}$ in $L^{2}$ which are relevant to Lemma 2.1.

Lemma 3.1. For $r$ in $] 3 / 2,2[$, we have

$$
\|\nabla a\|_{L^{r}} \lesssim\left\|\nabla a_{\frac{r}{2}}\right\|_{L^{2}}\left\|a_{\frac{r}{2}}\right\|_{L^{2}}^{\frac{2}{r}-1} .
$$

Moreover, for $s$ in $[-3 \alpha(r), 1-\alpha(r)]$, we have

$$
\|a\|_{\dot{H}^{s}} \leq C\left\|a_{\frac{r}{2}}\right\|_{L^{2}}^{1-\alpha(r)-s}\left\|\nabla a \frac{r}{2}\right\|_{L^{2}}^{3 \alpha(r)+s} .
$$

Proof. Notice that due to (2.9),

$$
\begin{aligned}
|\nabla a| & =\frac{2}{r}\left|\nabla a_{\frac{r}{2}}\right||a|^{1-\frac{r}{2}} \\
& =\frac{2}{r}\left|\nabla a_{\frac{r}{2}}\right|\left|a_{\frac{r}{2}}\right|^{\frac{2}{r}-1}
\end{aligned}
$$

then we get (3.1) by using Hölder inequality. The dual Sobolev inequality claims that

$$
\|a\|_{\dot{H}^{-3 \alpha(r)}} \leq C\|a\|_{L^{r}}=C\left\|a_{\frac{r}{2}}\right\|_{L^{2}}^{\frac{2}{r}} .
$$


Moreover, using again that $|\nabla a|=\frac{2}{r}\left|\nabla a_{\frac{r}{2}}\right|\left|a_{\frac{r}{2}}\right|^{\frac{2}{r}-1}$, Hölder inequality implies that

$$
\begin{aligned}
\|\nabla a\|_{L^{\frac{3 r}{1+r}}} & \leq \frac{2}{r}\left\|\nabla a_{\frac{r}{2}}\right\|_{L^{2}}\left\|a_{\frac{r}{2}}\right\|_{L^{6}}^{\frac{2}{r}-1} \\
& \lesssim\left\|\nabla a_{\frac{r}{2}}\right\|_{L^{2}}^{\frac{2}{r}} .
\end{aligned}
$$

Since $r<2$, we have $\frac{3 r}{1+r}<2$. Then Theorem 2.40 of [1] ensures that $L^{\frac{3 r}{1+r}} \hookrightarrow \dot{B}_{\frac{3 r}{1+r}, 2}^{0}$, which along with Bernstein's inequality implies

$$
\|a\|_{\dot{H}^{1-\alpha(r)}} \lesssim\|\nabla a\|_{\dot{B}_{\frac{3 r}{1+r}, 2}^{0}} \lesssim\left\|\nabla a_{\frac{r}{2}}\right\|_{L^{2}}^{\frac{2}{r}}
$$

from which and (3.3), we conclude the proof of (3.2) and hence the lemma by using interpolation inequality between $\dot{H}^{s} \mathrm{~S}$ obolev spaces.

As we shall use the anisotropic Littlewood-Paley theory, we recall the functional space framework we are going to use in this section. As in [3], [4], [8] and [5], the definitions of the spaces we are going to work with require anisotropic dyadic decomposition of the Fourier variables. Let us recall from [1] that

$$
\begin{aligned}
\Delta_{k}^{\mathrm{h}} a & =\mathcal{F}^{-1}\left(\varphi\left(2^{-k}\left|\xi_{\mathrm{h}}\right|\right) \widehat{a}\right), & \Delta_{\ell}^{\mathrm{v}} a & =\mathcal{F}^{-1}\left(\varphi\left(2^{-\ell}\left|\xi_{3}\right|\right) \widehat{a}\right), \\
S_{k}^{\mathrm{h}} a & =\mathcal{F}^{-1}\left(\chi\left(2^{-k}\left|\xi_{\mathrm{h}}\right|\right) \widehat{a}\right), & S_{\ell}^{\mathrm{v}} a & =\mathcal{F}^{-1}\left(\chi\left(2^{-\ell}\left|\xi_{3}\right|\right) \widehat{a}\right) \quad \text { and } \\
\Delta_{j} a & =\mathcal{F}^{-1}\left(\varphi\left(2^{-j}|\xi|\right) \widehat{a}\right), & S_{j} a & =\mathcal{F}^{-1}\left(\chi\left(2^{-j}|\xi|\right) \widehat{a}\right),
\end{aligned}
$$

where $\xi_{\mathrm{h}}=\left(\xi_{1}, \xi_{2}\right), \mathcal{F} a$ and $\widehat{a}$ denote the Fourier transform of the distribution $a, \chi(\tau)$ and $\varphi(\tau)$ are smooth functions such that

$$
\begin{aligned}
& \text { Supp } \varphi \subset\left\{\tau \in \mathbb{R} / \frac{3}{4} \leq|\tau| \leq \frac{8}{3}\right\} \quad \text { and } \quad \forall \tau>0, \sum_{j \in \mathbb{Z}} \varphi\left(2^{-j} \tau\right)=1 \text {, } \\
& \text { Supp } \chi \subset\left\{\tau \in \mathbb{R} / \quad|\tau| \leq \frac{4}{3}\right\} \quad \text { and } \quad \chi(\tau)+\sum_{j \geq 0} \varphi\left(2^{-j} \tau\right)=1 \text {. }
\end{aligned}
$$

Definition 3.1. Let $(p, r)$ be in $[1,+\infty]^{2}$ and $s$ in $\mathbb{R}$. Let us consider $u$ in $\mathcal{S}_{h}^{\prime}\left(\mathbb{R}^{3}\right)$, which means that $u$ is in $\mathcal{S}^{\prime}\left(\mathbb{R}^{3}\right)$ and satisfies $\lim _{j \rightarrow-\infty}\left\|S_{j} u\right\|_{L^{\infty}}=0$. We set

$$
\|u\|_{\dot{B}_{p, r}^{s}} \stackrel{\text { def }}{=}\left\|\left(2^{j s}\left\|\Delta_{j} u\right\|_{L^{p}}\right)_{j}\right\|_{\ell^{r}(\mathbb{Z})} .
$$

- For $s<\frac{3}{p}$ (or $s=\frac{3}{p}$ if $r=1$ ), we define $\dot{B}_{p, r}^{s}\left(\mathbb{R}^{3}\right) \stackrel{\text { def }}{=}\left\{u \in \mathcal{S}_{h}^{\prime}\left(\mathbb{R}^{3}\right) \mid\|u\|_{\dot{B}_{p, r}^{s}}<\infty\right\}$.

- If $k$ is a positive integer and if $\frac{3}{p}+k \leq s<\frac{3}{p}+k+1$ (or $s=\frac{3}{p}+k+1$ if $r=1$ ), then we define $\dot{B}_{p, r}^{s}\left(\mathbb{R}^{3}\right)$ as the subset of distributions $u$ in $\mathcal{S}_{h}^{\prime}\left(\mathbb{R}^{3}\right)$ such that $\partial^{\beta} u$ belongs to $\dot{B}_{p, r}^{s-k}\left(\mathbb{R}^{3}\right)$ whenever $|\beta|=k$.

We remark that in the particular case when $p=r=2, \dot{B}_{p, r}^{s}$ coincides with the classical homogeneous Sobolev spaces $\dot{H}^{s}$. Likewise, we can also define Besov spaces in the inhomogeneous context (see [1] for instance).

The description of the regularity of $\omega_{r-1}$ in terms of Besov spaces will be useful. This is done thanks to the following lemma (see [5], Lemma 5.1). 
Lemma 3.2. Let $(s, \alpha)$ be in $] 0,1\left[^{2}\right.$ and $(p, q)$ in $[1, \infty]^{2}$. We consider a function $G$ from $\mathbb{R}$ to $\mathbb{R}$ which is Hölderian of exponent $\alpha$. Then for any $a$ in the Besov space $\dot{B}_{p, q}^{s}$, one has

$$
\|G(a)\|_{\dot{B}_{\frac{p}{\alpha}, \frac{q}{\alpha}}^{\alpha s}} \lesssim\|G\|_{C^{\alpha}}\left(\|a\|_{\dot{B}_{p, q}^{s}}\right)^{\alpha} \quad \text { with } \quad\|G\|_{C^{\alpha}} \stackrel{\text { def }}{=} \sup _{r \neq r^{\prime}} \frac{\left|G(r)-G\left(r^{\prime}\right)\right|}{\left|r-r^{\prime}\right|^{\alpha}} .
$$

Similar to Definition 3.1, we can also define the homogeneous anisotropic Besov space.

Definition 3.2. Let us define the space $\left(\dot{B}_{p, q_{1}}^{s_{1}}\right)_{\mathrm{h}}\left(\dot{B}_{p, q_{2}}^{s_{2}}\right)_{\mathrm{v}}$ as the space of distribution in $\mathcal{S}_{h}^{\prime}$ such that

$$
\|u\|_{\left(\dot{B}_{p, q_{1}}^{s_{1}}\right)_{\mathrm{h}}\left(\dot{B}_{p, q_{2}}^{s_{2}}\right)_{\mathrm{v}}} \stackrel{\text { def }}{=}\left(\sum_{k \in \mathbb{Z}} 2^{q_{1} k s_{1}}\left(\sum_{\ell \in \mathbb{Z}} 2^{q_{2} \ell s_{2}}\left\|\Delta_{k}^{\mathrm{h}} \Delta_{\ell}^{\mathrm{v}} u\right\|_{L^{p}}^{q_{2}}\right)^{q_{1} / q_{2}}\right)^{1 / q_{1}}
$$

is finite.

We remark that when $p=q_{1}=q_{2}=2$, the anisotropic Besov space $\left(\dot{B}_{p, q_{1}}^{s_{1}}\right)_{\mathrm{h}}\left(\dot{B}_{p, q_{2}}^{s_{2}}\right)_{\mathrm{v}}$ coincides with the classical homogeneous anisotropic Sobolev space $\dot{H}^{s_{1}, s_{2}}$ and thus the space $\left(\dot{B}_{2,2}^{-3 \alpha(r)+\theta}\right)_{\mathrm{h}}\left(\dot{B}_{2,2}^{-\theta}\right)_{\mathrm{v}}$ is the space $\mathcal{H}^{\theta, r}$ defined in Definition 2.1. Let us also remark that in the case when $q_{1}$ is different from $q_{2}$, the order of summation is important.

For the convenience of the readers, we recall the following anisotropic Bernstein type lemma from $[4,8]$ :

Lemma 3.3. Let $\mathcal{B}_{h}$ (resp. $\mathcal{B}_{v}$ ) a ball of $\mathbb{R}_{h}^{2}$ (resp. $\mathbb{R}_{v}$ ), and $\mathcal{C}_{h}$ (resp. $\mathcal{C}_{v}$ ) a ring of $\mathbb{R}_{h}^{2}$ (resp. $\mathbb{R}_{v}$ ); let $1 \leq p_{2} \leq p_{1} \leq \infty$ and $1 \leq q_{2} \leq q_{1} \leq \infty$. Then there holds:

If the support of $\widehat{a}$ is included in $2^{k} \mathcal{B}_{h}$, then

$$
\left\|\partial_{x_{\mathrm{h}}}^{\alpha} a\right\|_{L_{\mathrm{h}}^{p_{1}}\left(L_{\mathrm{v}}^{q_{1}}\right)} \lesssim 2^{k\left(|\alpha|+2\left(1 / p_{2}-1 / p_{1}\right)\right)}\|a\|_{L_{\mathrm{h}}^{p_{2}\left(L_{\mathrm{v}}^{q_{1}}\right)}} .
$$

If the support of $\widehat{a}$ is included in $2^{\ell} \mathcal{B}_{v}$, then

$$
\left\|\partial_{x_{3}}^{\beta} a\right\|_{L_{\mathrm{h}}^{p_{1}\left(L_{\mathrm{v}}^{q_{1}}\right)}} \lesssim 2^{\ell\left(\beta+\left(1 / q_{2}-1 / q_{1}\right)\right)}\|a\|_{L_{\mathrm{h}}^{p_{1}}\left(L_{\mathrm{v}}^{q_{2}}\right)} .
$$

If the support of $\widehat{a}$ is included in $2^{k} \mathcal{C}_{h}$, then

$$
\|a\|_{L_{\mathrm{h}}^{p_{1}}\left(L_{\mathrm{v}}^{q_{1}}\right)} \lesssim 2^{-k N} \sup _{|\alpha|=N}\left\|\partial_{x_{\mathrm{h}}}^{\alpha} a\right\|_{L_{\mathrm{h}}^{p_{1}}\left(L_{\mathrm{v}}^{q_{1}}\right)} .
$$

If the support of $\widehat{a}$ is included in $2^{\ell} \mathcal{C}_{v}$, then

$$
\|a\|_{L_{\mathrm{h}}^{p_{1}\left(L_{\mathrm{v}}^{q_{1}}\right)}} \lesssim 2^{-\ell N}\left\|\partial_{x_{3}}^{N} a\right\|_{L_{\mathrm{h}}^{p_{1}}\left(L_{\mathrm{v}}^{q_{1}}\right)} .
$$

As a corollary of Lemma 3.3, we have the following inequality, if $1 \leq p_{2} \leq p_{1}$,

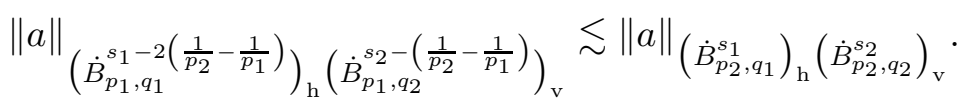

To consider the product of a distribution in the isotropic Besov space with a distribution in the anisotropic Besov space, we need the following result which allows to embed isotropic Besov spaces into the anisotropic ones.

Lemma 3.4 (Lemma 4.2 of [5]). Let $s$ be a positive real number and $(p, q)$ in $[1, \infty]$ with $p$ greater than or equal to $q$. Then one has

$$
\|a\|_{L_{\mathrm{h}}^{p}\left(\left(\dot{B}_{p, q}^{s}\right)_{\mathrm{v}}\right)} \lesssim\|a\|_{\dot{B}_{p, q}^{s}} .
$$


Lemma 3.5 (Lemma 4.3 of [5]). For any $s$ positive and any $\theta$ in $] 0, s[$, we have

$$
\|f\|_{\left(\dot{B}_{p, q}^{s-\theta}\right)_{\mathrm{h}}\left(\dot{B}_{p, 1}^{\theta}\right)_{\mathrm{v}}} \lesssim\|f\|_{\dot{B}_{p, q}^{s}} .
$$

One of the main motivation of using anisotropic Besov space is the proof of the following Proposition 3.1, which extends $r=3 / 2$ in [5] to general $r$ in ]3/2,2[.

Lemma 3.6. Let us consider $\theta$ in $] 0,3 \alpha(r)[$ and $\beta$ in $] 0,1 / 2[$. Then we have

$$
\|a\|_{\left(\dot{B}_{2,1}^{0}\right)_{\mathrm{h}}\left(\dot{B}_{2,1}^{1-3 \alpha(r)-\beta}\right)_{\mathrm{v}}} \lesssim\|a\|_{\mathcal{H}^{\theta, r}}^{\beta}\|\nabla a\|_{\mathcal{H}^{\theta, r}}^{1-\beta} .
$$

Proof. By definition of $\|\cdot\|_{\left(\dot{B}_{2,1}^{0}\right)_{\mathrm{h}}\left(\dot{B}_{2,1}^{1-3 \alpha(r)-\beta}\right)_{\mathrm{v}}}$, we have

$$
\begin{aligned}
\|a\|_{\left(\dot{B}_{2,1}^{0}\right)_{\mathrm{h}}\left(\dot{B}_{2,1}^{1-3 \alpha(r)-\beta}\right)_{\mathrm{v}}} & =H_{L}(a)+V_{L}(a) \text { with } \\
H_{L}(a) & \stackrel{\text { def }}{=} \sum_{k \leq \ell}\left\|\Delta_{k}^{\mathrm{h}} \Delta_{\ell}^{\mathrm{v}} a\right\|_{L^{2}} 2^{\ell(1-3 \alpha(r)-\beta)} \text { and } \\
V_{L}(a) & \stackrel{\text { def }}{=} \sum_{k>\ell}\left\|\Delta_{k}^{\mathrm{h}} \Delta_{\ell}^{\mathrm{v}} a\right\|_{L^{2}} 2^{\ell(1-3 \alpha(r)-\beta)} .
\end{aligned}
$$

In order to estimate $H_{L}(a)$, we classically estimate differently high and low vertical frequencies which are here the dominant ones. Using Lemma 3.3, we write that for any $N$ in $\mathbb{Z}$,

$$
H_{L}(a) \lesssim \sum_{k \leq \ell \leq N}\left\|\Delta_{k}^{h} \Delta_{\ell}^{\mathrm{v}} a\right\|_{L^{2}} 2^{\ell(1-3 \alpha(r)-\beta)}+\sum_{\substack{k \leq \ell \\ \ell>N}}\left\|\Delta_{k}^{h} \Delta_{\ell}^{\mathrm{v}} \partial_{3} a\right\|_{L^{2}} 2^{-\ell(3 \alpha(r)+\beta)} .
$$

By definition of the norm of $\mathcal{H}^{\theta, r}$, we get

$$
H_{L}(a) \lesssim\|a\|_{\mathcal{H}^{\theta, r}} \sum_{k \leq \ell \leq N} 2^{k(3 \alpha(r)-\theta)} 2^{\ell(1-3 \alpha(r)-\beta+\theta)}+\left\|\partial_{3} a\right\|_{\mathcal{H}^{\theta, r}} \sum_{\substack{k \leq \ell \\ \ell>N}} 2^{k(3 \alpha(r)-\theta)} 2^{-\ell(3 \alpha(r)+\beta-\theta)} .
$$

The hypothesis on $(\beta, \theta)$ imply that

$$
\begin{aligned}
H_{L}(a) & \lesssim\|a\|_{\mathcal{H}^{\theta, r}} \sum_{\ell \leq N} 2^{\ell(1-\beta)}+\left\|\partial_{3} a\right\|_{\mathcal{H}^{\theta, r}} \sum_{\ell>N} 2^{-\ell \beta} \\
& \lesssim\|a\|_{\mathcal{H}^{\theta, r}} 2^{N(1-\beta)}+\left\|\partial_{3} a\right\|_{\mathcal{H}^{\theta, r}} 2^{-N \beta} .
\end{aligned}
$$

Choosing $N$ such that $2^{N} \sim \frac{\left\|\partial_{3} a\right\|_{\mathcal{H}^{\theta, r}}}{\|a\|_{\mathcal{H}^{\theta, r}}}$ gives

$$
H_{L}(a) \lesssim\|a\|_{\mathcal{H}^{\theta, r}}^{\beta}\left\|\partial_{3} a\right\|_{\mathcal{H}^{\theta, r}}^{1-\beta} .
$$

The term $V_{L}(a)$ is estimated along the same lines. In fact, we get, by using again Lemma 3.3, that

$$
\begin{gathered}
V_{L}(a) \lesssim \sum_{\ell<k \leq N}\left\|\Delta_{k}^{h} \Delta_{\ell}^{\mathrm{v}} a\right\|_{L^{2}} 2^{\ell(1-3 \alpha(r)-\beta)}+\sum_{\substack{\ell<k \\
k>N \\
k}}\left\|\Delta_{k}^{h} \Delta_{\ell}^{\mathrm{v}} \nabla_{\mathrm{h}} a\right\|_{L^{2}} 2^{\ell(1-3 \alpha(r)-\beta)} 2^{-k} \\
\lesssim\|a\|_{\mathcal{H}^{\theta, r}} \sum_{\substack{\ell<k \leq N\\
}} 2^{k(3 \alpha(r)-\theta)} 2^{\ell(1-3 \alpha(r)-\beta+\theta)} \\
+\left\|\nabla_{\mathrm{h}} a\right\|_{\mathcal{H}^{\theta, r}} \sum_{\substack{\ell \leq k \\
k>N}} 2^{-k(1-3 \alpha(r)+\theta)} 2^{\ell(1-3 \alpha(r)-\beta+\theta)} \\
\lesssim\|a\|_{\mathcal{H}^{\theta, r}} 2^{N(1-\beta)}+\left\|\nabla_{\mathrm{h}} a\right\|_{\mathcal{H}^{\theta, r}} 2^{-N \beta} .
\end{gathered}
$$


Choosing $N$ such that $2^{N} \sim \frac{\left\|\nabla_{\mathrm{h}} a\right\|_{\mathcal{H}^{\theta, r}}}{\|a\|_{\mathcal{H}^{\theta, r}}}$ yields

$$
V_{L}(a) \lesssim\|a\|_{\mathcal{H}^{\theta, r}}^{\beta}\left\|\nabla_{\mathrm{h}} a\right\|_{\mathcal{H}^{\theta, r}}^{1-\beta} .
$$

Together with (3.7) and (3.8), this ensures the lemma.

Proposition 3.1. Let $v$ be a divergence free vector field. Let us consider $\theta$ in $] 0,3 \alpha(r)[$ and $\beta$ in $] 0,1 / 2[$. Then we have

$$
\left\|v^{\mathrm{h}}\right\|_{\left(\dot{B}_{2,1}^{1}\right)_{\mathrm{h}}\left(\dot{B}_{2,1}^{1-3 \alpha(r)-\beta}\right)_{\mathrm{v}}} \lesssim\left\|\omega_{\frac{r}{2}}\right\|_{L^{2}}^{2 \alpha(r)+\beta}\left\|\nabla \omega_{\frac{r}{2}}\right\|_{\mathcal{H}^{\theta, r}}^{1-\beta}+\left\|\partial_{3} v^{3}\right\|_{L^{2}}^{\beta}\left\|\nabla \partial_{3} v^{3}\right\|_{\mathcal{H}^{\theta, r}}^{1-\beta} .
$$

Proof. Using horizontal Biot-Savart law (2.4) and Lemma 3.3, we have

$$
\left\|v^{\mathrm{h}}\right\|_{\left(\dot{B}_{2,1}^{1}\right)_{\mathrm{h}}}\left(\dot{B}_{2,1}^{1-3 \alpha(r)-\beta}\right)_{\mathrm{v}} \lesssim\|\omega\|_{\left(\dot{B}_{2,1}^{0}\right)_{\mathrm{h}}\left(\dot{B}_{2,1}^{1-3 \alpha(r)-\beta}\right)_{\mathrm{v}}}+\left\|\partial r t i a l_{3} v^{3}\right\|_{\left(\dot{B}_{2,1}^{0}\right)_{\mathrm{h}}}\left(\dot{B}_{2,1}^{1-3 \alpha(r)-\beta}\right)_{\mathrm{v}}
$$

Applying Lemma 3.3 and Lemma 3.5 gives

$$
\begin{aligned}
\|\omega\|_{\left(\dot{B}_{2,1}^{0}\right)_{\mathrm{h}}\left(\dot{B}_{2,1}^{1-3 \alpha(r)-\beta}\right)_{\mathrm{v}}} & \lesssim\|\omega\|_{\left(\dot{B}_{\frac{3 r}{1+r}, 1}^{\frac{2}{3} \alpha(r)}\right)_{\mathrm{h}}}\left(\dot{B}_{\frac{3 r}{1+r}, 1}^{1-\frac{8}{3} \alpha(r)-\beta}\right)_{\mathrm{v}} \\
& \lesssim\|\omega\|_{\dot{B}_{\frac{3 r}{1+r}, 1}^{1-2 \alpha(r)-\beta}}
\end{aligned}
$$

Now let us estimate $\|\omega\|_{\dot{B}_{\frac{3 r}{1+r}, 1}^{s}}$ in terms of $\left\|\omega_{\frac{r}{2}}\right\|_{L^{2}}$ and $\left\|\nabla \omega_{\frac{r}{2}}\right\|_{L^{2}}$. For $s$ in $]-2 \alpha(r), 1[$ and any positive integer $N$, which we shall choose hereafter, we write that

$$
\begin{aligned}
\|\omega\|_{\dot{B}_{\frac{3 r}{1+r}, 1}^{s}} & =\sum_{j \leq N} 2^{j s}\left\|\Delta_{j} \omega\right\|_{L^{\frac{3 r}{1+r}}}+\sum_{j>N} 2^{j s}\left\|\Delta_{j} \omega\right\|_{L^{\frac{3 r}{1+r}}} \\
& \lesssim \sum_{j \leq N} 2^{j(s+2 \alpha(r))}\left\|\Delta_{j} \omega\right\|_{L^{r}}+\sum_{j>N} 2^{j(s-1)}\left\|\Delta_{j} \nabla \omega\right\|_{L^{\frac{3 r}{1+r}}} \\
& \lesssim 2^{N(s+2 \alpha(r))}\|\omega\|_{L^{r}}+2^{N(s-1)}\|\nabla \omega\|_{L^{\frac{3 r}{1+r}}}
\end{aligned}
$$

Choosing $N$ such that $2^{N} \sim\left(\frac{\|\nabla \omega\|}{\|\omega\|_{L^{r}} \frac{3 r}{1+r}}\right)^{\frac{1}{1+2 \alpha(r)}}$ yields

$$
\|\omega\|_{\dot{B}_{\frac{3 r}{1+r}, 1}} \lesssim\|\omega\|_{L^{r}}^{\frac{1-s}{1+2 \alpha(r)}}\|\nabla \omega\|_{L^{\frac{3 r}{1+r}}}^{\frac{s+2 \alpha(r)}{1+2 \alpha(r)}} .
$$

Due to $(1.2), 1+2 \alpha(r)=\frac{2}{r}$, then using the above inequality with $\left.s=1-2 \alpha(r)-\beta \in\right]-2 \alpha(r), 1[$, (3.10), and (3.4) gives

$$
\begin{aligned}
\|\omega\|_{\left(B_{2,1}^{0}\right)_{\mathrm{h}}\left(B_{2,1}^{1-3 \alpha(r)-\beta}\right)_{\mathrm{v}}} & \lesssim\|\omega\|_{L^{r}}^{\frac{r}{2}(2 \alpha(r)+\beta)}\|\nabla \omega\|_{L^{\frac{3 r}{1+r}}}^{\frac{r}{2}(1-\beta)} \\
& \lesssim\left\|\omega_{\frac{r}{2}}\right\|_{L^{2}}^{2 \alpha(r)+\beta}\left\|\nabla \omega_{\frac{r}{2}}\right\|_{L^{2}}^{1-\beta}
\end{aligned}
$$

The application of Lemma 3.6 together with (3.9) and (3.11) leads to Proposition 3.1.

To study product laws between distributions in the anisotropic Besov spaces, we need to modify the isotropic para-differential decomposition of Bony [2] to the setting of anisotropic 
version. We first recall the isotropic para-differential decomposition from [2]: let $a$ and $b$ be in $\mathcal{S}^{\prime}\left(\mathbb{R}^{3}\right)$,

$$
\begin{aligned}
& a b=T(a, b)+\bar{T}(a, b)+R(a, b) \quad \text { with } \\
& T(a, b)=\sum_{j \in \mathbb{Z}} S_{j-1} a \Delta_{j} b, \quad \bar{T}(a, b)=T(b, a), \quad \text { and } \\
& R(a, b)=\sum_{j \in \mathbb{Z}} \Delta_{j} a \tilde{\Delta}_{j} b, \quad \text { with } \quad \tilde{\Delta}_{j} b=\sum_{\ell=j-1}^{j+1} \Delta_{\ell} a .
\end{aligned}
$$

Sometimes we shall use Bony's decomposition for both horizontal and vertical variables simultaneously.

Finally let us recall the following product laws in the anisotropic Besov spaces from [5]:

Lemma 3.7 (Lemma 4.5 of [5]). Let $q \geq 1, p_{1} \geq p_{2} \geq 1$ with $1 / p_{1}+1 / p_{2} \leq 1$, and $s_{1}<2 / p_{1}$, $s_{2}<2 / p_{2}$ (resp. $s_{1} \leq 2 / p_{1}, s_{2} \leq 2 / p_{2}$ if $q=1$ ) with $s_{1}+s_{2}>0$. Let $\sigma_{1}<1 / p_{1}, \sigma_{2}<1 / p_{2}$ (resp. $\sigma_{1} \leq 1 / p_{1}, \sigma_{2} \leq 1 / p_{2}$ if $q=1$ ) with $\sigma_{1}+\sigma_{2}>0$. Then for $a$ in $\left(\dot{B}_{p_{1}, q}^{s_{1}}\right)_{\mathrm{h}}\left(\dot{B}_{p_{1}, q}^{\sigma_{1}}\right)_{\mathrm{v}}$ and $b$ in $\left(\dot{B}_{p_{2}, q}^{s_{2}}\right)_{\mathrm{h}}\left(\dot{B}_{p_{2}, q}^{\sigma_{2}}\right)_{\mathrm{v}}$, the product ab belongs to $\left(\dot{B}_{p_{1}, q}^{s_{1}+s_{2}-2 / p_{2}}\right)_{\mathrm{h}}\left(\dot{B}_{p_{1}, q}^{\sigma_{1}+\sigma_{2}-1 / p_{2}}\right)_{\mathrm{v}}$, and

$$
\|a b\|_{\left(\dot{B}_{p_{1}, q}^{s_{1}+s_{2}-2 / p_{2}}\right)_{\mathrm{h}}\left(\dot{B}_{p_{1}, q}^{\sigma_{1}+\sigma_{2}-1 / p_{2}}\right)_{\mathrm{v}}} \lesssim\|a\|_{\left(\dot{B}_{p_{1}, q}^{s_{1}}\right)_{\mathrm{h}}}\left(\dot{B}_{p_{1}, q}^{\sigma_{1}}\right)_{\mathrm{v}}\|b\|_{\left(\dot{B}_{p_{2}, q}^{s_{2}}\right)_{\mathrm{h}}}\left(\dot{B}_{p_{2}, q}^{\sigma_{2}}\right)_{\mathrm{v}} \cdot
$$

\section{Proof of the estimate for the horizontal vorticity}

The purpose of this section to present the proof of Proposition 2.1. Let us recall the first equation of our reformulation $(\widetilde{N S})$ of the incompressible Navier-Stokes equation which is

$$
\partial_{t} \omega+v \cdot \nabla \omega-\Delta \omega=\partial_{3} v^{3} \omega+\partial_{2} v^{3} \partial_{3} v^{1}-\partial_{1} v^{3} \partial_{3} v^{2}
$$

As already explained in the second section, we decompose the right-hand side term as a sum of three terms. Hence by virtue of (2.6), we obtain

$$
\begin{aligned}
& \frac{1}{r}\left\|\omega_{\frac{r}{2}}(t)\right\|_{L^{2}}^{2}+\frac{4(r-1)}{r^{2}} \int_{0}^{t}\left\|\nabla \omega_{\frac{r}{2}}\left(t^{\prime}\right)\right\|_{L^{2}}^{2} d t^{\prime}=\frac{1}{r}\left\|\left|\omega_{0}\right|^{\frac{r}{2}}\right\|_{L^{2}}^{2}+\sum_{\ell=1}^{3} F_{\ell}(t) \quad \text { with } \\
& F_{1}(t) \stackrel{\text { def }}{=} \int_{0}^{t} \int_{\mathbb{R}^{3}} \partial_{3} v^{3}|\omega|^{r} d x d t^{\prime}, \\
& F_{2}(t) \stackrel{\text { def }}{=} \int_{0}^{t} \int_{\mathbb{R}^{3}}\left(\partial_{2} v^{3} \partial_{3} v_{\text {curl }}^{1}-\partial_{1} v^{3} \partial_{3} v_{\text {curl }}^{2}\right) \omega_{r-1} d x d t^{\prime} \quad \text { and } \\
& F_{3}(t) \stackrel{\text { def }}{=} \int_{0}^{t} \int_{\mathbb{R}^{3}}\left(\partial_{2} v^{3} \partial_{3} v_{\text {div }}^{1}-\partial_{1} v^{3} \partial_{3} v_{\text {div }}^{2}\right) \omega_{r-1} d x d t^{\prime},
\end{aligned}
$$

where $v_{\text {curl }}^{\mathrm{h}}$ (resp. $v_{\text {div }}^{\mathrm{h}}$ ) corresponds to the horizontal divergence free (resp. curl free) part of the horizontal vector $v^{\mathrm{h}}=\left(v^{1}, v^{2}\right)$, which is given by $(2.4)$, and where $\omega_{r-1} \stackrel{\text { def }}{=}|\omega|^{r-2} \omega$.

Let us start with the easiest term $F_{1}$. We first get, by using integration by parts, that

$$
\begin{aligned}
\left|F_{1}(t)\right| & \leq r \int_{0}^{t} \int_{\mathbb{R}^{3}}\left|v^{3}\left(t^{\prime}, x\right)\right|\left|\partial_{3} \omega\left(t^{\prime}, x\right)\right|\left|\omega\left(t^{\prime}, x\right)\right|^{r-1} d x d t^{\prime} \\
& \leq r \int_{0}^{t} \int_{\mathbb{R}^{3}}\left|v^{3}\left(t^{\prime}, x\right)\right|\left|\partial_{3} \omega\left(t^{\prime}, x\right)\right|\left|\omega_{\frac{r}{2}}\left(t^{\prime}, x\right)\right|^{\frac{2}{r^{\prime}}} d x d t^{\prime} .
\end{aligned}
$$


Using that

$$
\frac{p-2}{3 p}+\frac{1}{r}+\frac{2 p r-3 p+2 r}{6 p(r-1)} \times \frac{2}{r^{\prime}}=1
$$

we apply Hölder inequality to get

$$
\left|F_{1}(t)\right| \leq r \int_{0}^{t}\left\|v^{3}\left(t^{\prime}\right)\right\|_{L^{\frac{3 p}{p-2}}}\left\|\partial_{3} \omega\left(t^{\prime}\right)\right\|_{L^{r}}\left\|\omega_{\frac{r}{2}}\left(t^{\prime}\right)\right\|_{L^{\frac{6 p(r-1)}{2 p r-3 p+2 r}}}^{\frac{2}{r^{\prime}}} d t^{\prime} .
$$

As $p$ is in $] 4, \frac{2 r}{2-r}\left[\right.$, we have that $r^{\prime} \frac{p-2}{2 p}$ belongs to $] 0,1[$. Then Sobolev embedding and interpolation inequality implies that

$$
\left\|\omega_{\frac{r}{2}}\left(t^{\prime}\right)\right\|_{L^{\frac{6 p(r-1)}{2 p r-3 p+2 r}}} \lesssim\left\|\omega_{\frac{r}{2}}\left(t^{\prime}\right)\right\|_{\dot{H}^{r^{\prime}} \frac{(p-2)}{2 p}} \lesssim\left\|\omega_{\frac{r}{2}}\left(t^{\prime}\right)\right\|_{L^{2}}^{\frac{2 r-p(2-r)}{2(r-1)}}\left\|\nabla \omega_{\frac{r}{2}}\left(t^{\prime}\right)\right\|_{L^{2}}^{r^{\prime} \frac{p-2}{2 p}}
$$

Using (3.1) of Lemma 3.1, this gives

$$
\left|F_{1}(t)\right| \lesssim \int_{0}^{t}\left\|v^{3}\left(t^{\prime}\right)\right\|_{\dot{H}^{\frac{1}{2}+\frac{2}{p}}}\left\|\partial_{3} \omega_{\frac{r}{2}}\left(t^{\prime}\right)\right\|_{L^{2}}\left\|\omega_{\frac{r}{2}}\left(t^{\prime}\right)\right\|_{L^{2}}^{\frac{2}{r}-1}\left\|\nabla \omega_{\frac{r}{2}}\left(t^{\prime}\right)\right\|_{L^{2}}^{1-\frac{2}{p}}\left\|\omega_{\frac{r}{2}}\left(t^{\prime}\right)\right\|_{L^{2}}^{1-2\left(\frac{1}{r}-\frac{1}{p}\right)} d t^{\prime} .
$$

Applying convex inequality, we obtain

$$
\begin{aligned}
\left|F_{1}(t)\right| & \lesssim \int_{0}^{t}\left\|v^{3}\left(t^{\prime}\right)\right\|_{\dot{H}^{\frac{1}{2}+\frac{2}{p}}}\left\|\omega_{\frac{r}{2}}\left(t^{\prime}\right)\right\|_{L^{2}}^{\frac{2}{p}}\left\|\nabla \omega_{\frac{r}{2}}\left(t^{\prime}\right)\right\|_{L^{2}}^{\frac{2}{p^{\prime}}} d t^{\prime} \\
& \leq \frac{r-1}{r^{2}} \int_{0}^{t}\left\|\nabla \omega_{\frac{r}{2}}\left(t^{\prime}\right)\right\|_{L^{2}}^{2} d t^{\prime}+C \int_{0}^{t}\left\|v^{3}\left(t^{\prime}\right)\right\|_{\dot{H}^{\frac{1}{2}+\frac{2}{p}}}^{p}\left\|\omega_{\frac{r}{2}}\left(t^{\prime}\right)\right\|_{L^{2}}^{2} d t^{\prime} .
\end{aligned}
$$

The other two terms in (4.1) require a refined way of the description of the regularity of $\omega_{\frac{r}{2}}$ and demand a detailed study of the anisotropic operator $\nabla_{\mathrm{h}} \Delta_{\mathrm{h}}^{-1}$ associated with the BiotSavart's law in horizontal variables. Now we first modify Lemma 5.2 of [5] to the following one.

Lemma 4.1. Let $\theta$ be in $] 0, \alpha(r)[$ for $\alpha(r)$ given by (1.2), and $\sigma, s$ be such that

$$
\sigma \in] \frac{r^{\prime}}{4}, 1\left[\text { and } s=\frac{1}{2}+1-\frac{2 \sigma}{r^{\prime}} .\right.
$$

Then we have

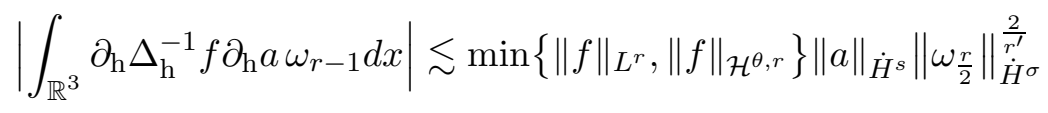

for $\mathcal{H}^{\theta, r}$ given by Definition 2.1.

Proof. Let us observe that $\omega_{r-1}=G\left(\omega_{\frac{r}{2}}\right)$ with $G(z) \stackrel{\text { def }}{=} z|z|^{-2 \alpha(r)}$. Using Lemma 3.2, we obtain

$$
\left\|\omega_{r-1}\right\|_{\dot{B}_{r^{\prime}, r^{\prime}}^{\frac{2 \sigma}{r^{\prime}}}} \lesssim\left\|\omega_{\frac{r}{2}}\right\|_{\dot{H}^{\sigma}}^{\frac{2}{r^{\prime}}}
$$

Let us study the product $\partial_{\mathrm{h}} a \omega_{r-1}$. Using Bony's decomposition (3.12) and the Leibnitz formula, we write

$$
\begin{aligned}
& \partial_{\mathrm{h}} a \omega_{r-1}=T\left(\partial_{\mathrm{h}} a, \omega_{r-1}\right)+R\left(\partial_{\mathrm{h}} a, \omega_{r-1}\right)+T\left(\omega_{r-1}, \partial_{\mathrm{h}} a\right) \\
&=\partial_{\mathrm{h}} T\left(\omega_{r-1}, a\right)+A(a, \omega) \text { with } \\
& A(a, \omega) \stackrel{\text { def }}{=} T\left(\partial_{\mathrm{h}} a, \omega_{r-1}\right)+R\left(\partial_{\mathrm{h}} a, \omega_{r-1}\right)-T\left(\partial_{\mathrm{h}} \omega_{r-1}, a\right) .
\end{aligned}
$$


W e first get, by using Lemma 3.3, that

$$
\begin{aligned}
\left\|\Delta_{j} T\left(\omega_{r-1}, a\right)\right\|_{L^{2}} & \lesssim \sum_{\left|j-j^{\prime}\right| \leq 4}\left\|S_{j^{\prime}-1} \omega_{r-1}\right\|_{L^{\infty}}\left\|\Delta_{j^{\prime}} a\right\|_{L^{2}} \\
& \lesssim \sum_{\left|j-j^{\prime}\right| \leq 4} 2^{j^{\prime}\left(\frac{3}{r^{\prime}}-\frac{2 \sigma}{r^{\prime}}\right)}\left\|\omega_{r-1}\right\|_{\dot{B}_{r^{\prime}, r^{\prime}}^{\frac{2 \sigma}{r^{\prime}}}} c_{j^{\prime}, 2} 2^{-j^{\prime} s}\|a\|_{\dot{H}^{s}} \\
& \lesssim c_{j, 2} 2^{-j\left(s-\frac{1}{r^{\prime}}(3-2 \sigma)\right)}\left\|\omega_{r-1}\right\|_{\dot{B}_{r^{\prime}, r^{\prime}}^{\frac{2 \sigma}{r^{\prime}}}}\|a\|_{\dot{H}^{s}} .
\end{aligned}
$$

Here and in what follows, we always denote $\left(c_{j, r}\right)_{j \in \mathbb{Z}}$ to be a generic element in the sphere of $\ell^{r}(\mathbb{Z})$. Then together with (4.4), the above inequality ensures that

$$
\left\|T\left(\omega_{r-1}, a\right)\right\|_{\dot{H}^{s-\frac{1}{r^{\prime}}(3-2 \sigma)}} \lesssim\|a\|_{\dot{H}^{s}}\left\|\omega_{\frac{r}{2}}\right\|_{\dot{H}^{\sigma}}^{\frac{2}{r^{\prime}}}
$$

Using that the operator $\partial_{\mathrm{h}}^{2} \Delta_{\mathrm{h}}^{-1}$ is a bounded Fourier multiplier and the dual Sobolev embed$\operatorname{ding} L^{r} \hookrightarrow \dot{H}^{-3 \alpha(r)}$, we get by taking $s=\frac{3}{2}-\frac{2 \sigma}{r^{\prime}}$ in (4.5) that

$$
\begin{aligned}
\left|\int_{\mathbb{R}^{3}} \partial_{\mathrm{h}} \Delta_{\mathrm{h}}^{-1} f \partial_{\mathrm{h}} T\left(\omega_{r-1}, a\right) d x\right| & =\left|\int_{\mathbb{R}^{3}} \partial_{\mathrm{h}}^{2} \Delta_{\mathrm{h}}^{-1} f T\left(\omega_{r-1}, a\right) d x\right| \\
& \leq\|f\|_{\dot{H}^{-3 \alpha(r)}}\left\|T\left(\omega_{r-1}, a\right)\right\|_{\dot{H}^{3 \alpha}(r)} \\
& \lesssim\|f\|_{L^{r}}\|a\|_{\dot{H}^{s}}\left\|\omega_{\frac{r}{2}}\right\|_{\dot{H}^{\sigma}}^{\frac{2}{r^{\prime}}} .
\end{aligned}
$$

In the case of the anisotropic norm, recalling that $\mathcal{H}^{\theta, r}=\dot{H}^{-3 \alpha(r)+\theta,-\theta}$, and using Lemma ??, we write

$$
\begin{aligned}
\left|\int_{\mathbb{R}^{3}} \operatorname{partial}_{\mathrm{h}}^{2} \Delta_{\mathrm{h}}^{-1} f T\left(\omega_{r-1}, a\right) d x\right| & \leq\|f\|_{\mathcal{H}^{\theta, r}}\left\|T\left(\omega_{r-1}, a\right)\right\|_{\dot{H}^{3 \alpha(r)-\theta, \theta}} \\
& \lesssim\|f\|_{\mathcal{H}^{\theta, r}}\left\|T\left(\omega_{r-1}, a\right)\right\|_{\dot{H}^{3 \alpha(r)}} \\
& \lesssim\|f\|_{\mathcal{H}^{\theta, r}}\|a\|_{\dot{H}^{s}}\left\|\omega_{\frac{r}{2}}\right\|_{\dot{H}^{\sigma}}^{\frac{2}{r^{\prime}}}
\end{aligned}
$$

Now let us take into account the anisotropy induced by the operator $\partial_{\mathrm{h}} \Delta_{\mathrm{h}}^{-1}$. HardyLittlewood-Sobolev inequality implies that $\partial_{\mathrm{h}} \Delta_{\mathrm{h}}^{-1} f$ belongs to $L_{\mathrm{v}}^{r}\left(L_{\mathrm{h}}^{\frac{2 r}{2-r}}\right)$ if $f$ is in $L^{r}$. So that it amounts to prove that $A(a, \omega)$ belongs to $L_{\mathrm{v}}^{r^{\prime}}\left(L_{\mathrm{h}}^{\frac{2 r}{3 r-2}}\right)$, which is simply an anisotropic Sobolev type embedding. Because of $s=\frac{1}{2}+1-\frac{2 \sigma}{r^{\prime}}<1$, we get, by using Lemma 3.3, Inequality (4.4) and Hölder inequality with 2 and $r^{\prime}$, that

$$
\begin{aligned}
\left\|\Delta_{j} T\left(\partial_{\mathrm{h}} a, \omega_{r-1}\right)\right\|_{L^{\frac{2 r}{3 r-2}}} & \lesssim \sum_{\left|j^{\prime}-j\right| \leq 4}\left\|S_{j^{\prime}-1} \partial_{\mathrm{h}} a\right\|_{L^{2}}\left\|\Delta_{j^{\prime}} \omega_{r-1}\right\|_{L^{r^{\prime}}} \\
& \lesssim \sum_{\left|j^{\prime}-j\right| \leq 4} c_{j^{\prime}, 2} c_{j^{\prime}, r^{\prime}} 2^{j^{\prime}(1-s)}\|a\|_{\dot{H}^{s}} 2^{-2 j^{\prime}} \frac{\sigma}{r^{\prime}}\left\|\omega_{\frac{r}{2}}\right\|_{\dot{H}^{\sigma}}^{\frac{2}{r^{\prime}}} \\
& \lesssim c_{j, \frac{2 r}{3 r-2}} 2^{-\frac{j}{2}}\|a\|_{\dot{H}^{s}}\left\|\omega_{\frac{r}{2}}\right\|_{\dot{H}^{\sigma}}^{\frac{2}{r^{\prime}}} .
\end{aligned}
$$


Along the same lines, we have

$$
\begin{aligned}
\left\|\Delta_{j} R\left(\partial_{\mathrm{h}} a, \omega_{r-1}\right)\right\|_{L^{\frac{2 r}{3 r-2}}} & \lesssim \sum_{j^{\prime} \geq j-3}\left\|\Delta_{j^{\prime}} \partial_{\mathrm{h}} a\right\|_{L^{2}}\left\|\widetilde{\Delta}_{j^{\prime}} \omega_{r-1}\right\|_{L^{r^{\prime}}} \\
& \lesssim \sum_{j^{\prime} \geq j-3} c_{j^{\prime}, 2} c_{j^{\prime}, r^{\prime}} 2^{j^{\prime}(1-s)}\|a\|_{\dot{H}^{s}} 2^{-2 j^{\prime} \frac{\sigma}{r^{\prime}}}\left\|\omega_{\frac{r}{2}}\right\|_{\dot{H}^{\sigma}}^{\frac{2}{r^{\prime}}} \\
& \lesssim c_{j, \frac{2 r}{3 r-2}} 2^{-\frac{j}{2}}\|a\|_{\dot{H}^{s}}\left\|\omega_{\frac{r}{2}}\right\|_{\dot{H}^{\sigma}}^{\frac{2}{r^{\prime}}},
\end{aligned}
$$

and

$$
\begin{aligned}
\left\|\Delta_{j} T\left(\partial_{\mathrm{h}} \omega_{r-1}, a\right)\right\|_{L^{\frac{2 r}{3 r-2}}} & \lesssim \sum_{\left|j^{\prime}-j\right| \leq 4}\left\|S_{j^{\prime}-1} \partial_{\mathrm{h}} \omega_{r-1}\right\|_{L^{r^{\prime}}}\left\|\widetilde{\Delta}_{j^{\prime}} a\right\|_{L^{2}} \\
& \lesssim c_{j, \frac{2 r}{3 r-2}} 2^{-\frac{j}{2}}\|a\|_{\dot{H}^{s}}\left\|\omega_{\frac{r}{2}}\right\|_{\dot{H}^{\sigma}}^{\frac{2}{r^{\prime}}}
\end{aligned}
$$

This leads to

$$
\|A(a, \omega)\|_{\frac{\dot{B}^{\frac{1}{2}} \frac{2 r}{3 r-2}, \frac{2 r}{3 r-2}}{}} \lesssim\|a\|_{\dot{H}^{s}}\left\|\omega_{\frac{r}{2}}\right\|_{\dot{H}^{\sigma}}^{\frac{2}{r^{\prime}}} .
$$

While it follows from Lemma 3.4 that

$$
\dot{B}_{\frac{2 r}{3 r-2}, \frac{2 r}{3 r-2}}^{\frac{1}{2}} \hookrightarrow L_{\mathrm{h}}^{\frac{2 r}{3 r-2}}\left(\left(\dot{B}_{\frac{1}{3 r-2}, \frac{2 r}{3 r-2}}^{\frac{1}{3 r}}\right)_{\mathrm{V}}\right) .
$$

Sobolev type embedding theorem (see for instance Theorem 2.40 of [1]) claims that

$$
\dot{B}_{\frac{2 r}{3 r-2}, \frac{2 r}{3 r-2}}^{\frac{1}{2}}(\mathbb{R}) \hookrightarrow \dot{B}_{r^{\prime}, 2}^{0}(\mathbb{R}) \hookrightarrow L^{r^{\prime}}(\mathbb{R}) .
$$

Moreover, since $r^{\prime}>\frac{2 r}{3 r-2}$, we have

$$
L_{\mathrm{h}}^{\frac{2 r}{3 r-2}}\left(L_{\mathrm{v}}^{r^{\prime}}\right) \hookrightarrow L_{\mathrm{v}}^{r^{\prime}}\left(L_{\mathrm{h}}^{\frac{2 r}{3 r-2}}\right) .
$$

As a consequence, by virtue of (4.8), we obtain

$$
\begin{aligned}
\left|\int_{\mathbb{R}^{3}} \partial_{\mathrm{h}} \Delta_{\mathrm{h}}^{-1} f A(a, \omega) d x\right| & \lesssim\left\|\partial_{\mathrm{h}} \Delta_{\mathrm{h}}^{-1} f\right\|_{L_{\mathrm{v}}^{r}\left(L_{\mathrm{h}}^{2-r}\right)}\|A(a, \omega)\|_{L_{\mathrm{v}}^{r^{\prime}}\left(L_{\mathrm{h}}^{\frac{2 r}{3 r-2}}\right)} \\
& \lesssim\|f\|_{L^{r}}\|A(a, \omega)\|_{\dot{B}^{\frac{1}{2}} \frac{2 r}{3 r-2}, \frac{2 r}{3 r-2}} \\
& \lesssim\|f\|_{L^{r}}\|a\|_{\dot{H}^{s}}\left\|\omega_{\frac{r}{2}}\right\|_{\dot{H}^{\sigma}}^{\frac{2}{r^{\prime}}},
\end{aligned}
$$

which together with (4.6) gives rise to

$$
\left|\int_{\mathbb{R}^{3}} \partial_{\mathrm{h}} \Delta_{\mathrm{h}}^{-1} f \partial_{\mathrm{h}} a \omega_{r-1} d x\right| \lesssim\|f\|_{L^{r}}\|a\|_{\dot{H}^{s}}\left\|\varnothing m_{\frac{r}{2}}\right\|_{\dot{H}^{\sigma}}^{\frac{2}{r^{\prime}}}
$$

In order to prove the remaining inequality of (4.3), we observe that

$$
\left\|\nabla_{\mathrm{h}} \Delta_{\mathrm{h}}^{-1} f\right\|_{\dot{H}^{1-3 \alpha(r)+\theta,-\theta}} \lesssim\|f\|_{\dot{H}^{-3 \alpha(r)+\theta,-\theta}}=\|f\|_{\mathcal{H}^{\theta, r}} .
$$

Thus thanks to (4.8), for $\theta$ given by the lemma, it amounts to prove that

$$
\dot{B}_{\frac{2 r}{3 r-2}, \frac{2 r}{3 r-2}}^{\frac{1}{2}} \hookrightarrow \dot{H}^{-1+3 \alpha(r)-\theta, \theta} .
$$


As a matter of fact, using Lemma 3.5 and Lemma 3.3, we have, for any $\gamma$ in $] 0,1 / 2[$,

$$
\begin{aligned}
\dot{B}_{\frac{2 r}{3 r-2}, \frac{1}{3 r-2}}^{\frac{1}{3 r-2}} & \hookrightarrow\left(\dot{B}_{\frac{12}{3 r-2}, \frac{2 r}{3 r-2}}^{\frac{1}{2}}\right)_{\mathrm{h}}\left(\dot{B}_{\frac{2 r}{3 r-2}, \frac{2 r}{3 r-2}}^{\gamma}\right)_{\mathrm{v}} \quad \text { and } \\
\left(\dot{B}_{\frac{2 r}{3 r-2}, \frac{2 r}{3 r-2}}^{\frac{1}{2}-\gamma}\right)_{\mathrm{h}}\left(\dot{B}_{\frac{2 r}{3 r-2}, \frac{2 r}{3 r-2}}^{\gamma}\right)_{\mathrm{v}} & \left.\hookrightarrow\left(\dot{B}_{2,2}^{\frac{1}{2}-\gamma-\frac{2}{r^{\prime}}}\right)_{\mathrm{h}}\left(\dot{B}_{2,2}^{\gamma-\frac{1}{r^{\prime}}}\right)\right)_{\mathrm{v}} \\
& \hookrightarrow \dot{H}^{-\gamma+\frac{2}{r}-\frac{3}{2}, \gamma-1+\frac{1}{r}}
\end{aligned}
$$

Let us choose $\theta \stackrel{\text { def }}{=} \gamma-\frac{1}{r^{\prime}}$. Then since $\theta<\alpha(r)$, we have $\gamma<\frac{1}{2}$, which ensures (4.9). This completes the proof of the lemma.

The estimate of $F_{2}(t)$ uses the Biot-Savart's law in the horizontal variables (namely (2.4)) and Lemma 4.1 with $f=\partial_{3} \omega, a=v^{3}$. This gives for any time $t<T^{\star}$ and $\sigma$ in $] \frac{r}{4(r-1)}, 1[$ that

$$
\begin{aligned}
& I_{\omega}(t) \stackrel{\text { def }}{=}\left|\int_{\mathbb{R}^{3}}\left(\partial_{2} v^{3}(t, x) \partial_{3} v_{\text {curl }}^{1}(t, x)-\partial_{1} v^{3}(t, x) \partial_{3} v_{\text {curl }}^{2}(t, x)\right) \omega_{r-1}(t, x) d x\right| \\
& \lesssim\left\|\partial_{3} \omega(t)\right\|_{L^{r}}\left\|v^{3}(t)\right\|_{\dot{H}^{\frac{3}{2}}-\frac{2 \sigma}{r^{\prime}}}\left\|\omega_{\frac{r}{2}}(t)\right\|_{\dot{H}^{\sigma}}^{\frac{2}{r^{\prime}}} .
\end{aligned}
$$

By virtue of (3.1) and of the interpolation inequalities between $L^{2}$ and $\dot{H}^{1}$, we thus obtain

$$
\begin{aligned}
& I_{\omega}(t) \lesssim\left\|v^{3}(t)\right\|_{\left.\dot{H}^{\frac{1}{2}+2\left(\frac{1}{2}-\frac{\sigma}{r^{\prime}}\right.}\right)}\left\|\omega_{\frac{r}{2}}(t)\right\|_{L^{2}}^{\frac{2}{r}-1}\left\|\nabla \omega_{\frac{r}{2}}(t)\right\|_{L^{2}} \\
& \times\left\|\omega_{\frac{r}{2}}(t)\right\|_{L^{2}}^{\frac{2}{r^{\prime}}(1-\sigma)}\left\|\nabla \omega_{\frac{r}{2}}(t)\right\|_{L^{2}}^{\frac{2 \sigma}{r^{\prime}}} \\
& \lesssim\left\|v^{3}(t)\right\|_{\dot{H}^{\frac{1}{2}+2\left(\frac{1}{2}-\frac{\sigma}{r^{\prime}}\right.} \|}\left\|\omega_{\frac{r}{2}}(t)\right\|_{L^{2}}^{2\left(\frac{1}{2}-\frac{\sigma}{r^{\prime}}\right)}\left\|\nabla \omega_{\frac{r}{2}}(t)\right\|_{L^{2}}^{2\left(\frac{1}{2}+\frac{\sigma}{r^{\prime}}\right)} \text {. }
\end{aligned}
$$

Choosing $\sigma=\frac{(p-2) r}{2 p(r-1)}$, which is between $\frac{r^{\prime}}{4}$ and 1 because $p$ is between 4 and $\frac{2 r}{2-r}$, gives

$$
I_{\omega}(t) \lesssim\left\|v^{3}(t)\right\|_{\dot{H}^{\frac{1}{2}+\frac{2}{p}}}\left\|\omega_{\frac{r}{2}}(t)\right\|_{L^{2}}^{\frac{2}{p}}\left\|\nabla \omega_{\frac{r}{2}}(t)\right\|_{L^{2}}^{2\left(1-\frac{1}{p}\right)}
$$

Then by using convexity inequality and time integration, we get

$$
\left|F_{2}(t)\right| \leq \frac{r-1}{r^{2}} \int_{0}^{t}\left\|\nabla \omega_{\frac{r}{2}}\left(t^{\prime}\right)\right\|_{L^{2}}^{2} d t^{\prime}+C \int_{0}^{t}\left\|v^{3}\left(t^{\prime}\right)\right\|_{\dot{H}^{\frac{1}{2}+\frac{2}{p}}}^{p}\left\|\omega_{\frac{r}{2}}\left(t^{\prime}\right)\right\|_{L^{2}}^{2} d t^{\prime} .
$$

In order to estimate $F_{3}(t)$, we write

$$
\begin{aligned}
F_{3}(t)=-\int_{0}^{t} \int_{\mathbb{R}^{3}}\left(\partial _ { 2 } v ^ { 3 } ( t ^ { \prime } , x ) \left(\partial_{1} \Delta_{\mathrm{h}}^{-1}\right.\right. & \left.\partial_{3}^{2} v^{3}\right)\left(t^{\prime}, x\right) \\
& \left.-\partial_{1} v^{3}\left(t^{\prime}, x\right)\left(\partial_{2} \Delta_{\mathrm{h}}^{-1} \partial_{3}^{2} v^{3}\right)\left(t^{\prime}, x\right)\right) \omega_{r-1}\left(t^{\prime}, x\right) d x d t^{\prime} .
\end{aligned}
$$


Since $\sigma=\frac{(p-2) r}{2 p(r-1)}, \frac{1}{p}=\frac{1}{2}-\frac{\sigma}{r^{\prime}}$, thanks to interpolation inequality between Sobolev spaces, we get, by applying Lemma 4.1 with $f=\partial_{3}^{2} v^{3}$ and $a=v^{3}$, that

$$
\begin{aligned}
\left|F_{3}(t)\right| & \lesssim \int_{0}^{t}\left\|\partial_{3}^{2} v^{3}\left(t^{\prime}\right)\right\|_{\mathcal{H}^{\theta, r}}\left\|v^{3}\left(t^{\prime}\right)\right\|_{\dot{H}^{\frac{3}{2}-\frac{2 \sigma}{r^{\prime}}}}\left\|\omega_{\frac{r}{2}}\left(t^{\prime}\right)\right\|_{\dot{H}^{\sigma}}^{\frac{2}{r^{\prime}}} d t^{\prime} \\
& \lesssim \int_{0}^{t}\left\|\partial_{3}^{2} v^{3}\left(t^{\prime}\right)\right\|_{\mathcal{H}^{\theta, r}}\left\|v^{3}\left(t^{\prime}\right)\right\|_{\dot{H}^{\frac{1}{2}+2\left(\frac{1}{2}-\frac{\sigma}{r^{\prime}}\right)}}\left\|\omega_{\frac{r}{2}}\left(t^{\prime}\right)\right\|_{L^{2}}^{\frac{2}{r^{\prime}}(1-\sigma)}\left\|\nabla \omega_{\frac{r}{2}}\left(t^{\prime}\right)\right\|_{L^{2}}^{\frac{2 \sigma}{r^{\prime}}} d t^{\prime} \\
& \lesssim \int_{0}^{t}\left\|\partial_{3}^{2} v^{3}\left(t^{\prime}\right)\right\|_{\mathcal{H}^{\theta, r}}\left\|v^{3}\left(t^{\prime}\right)\right\|_{\dot{H}^{\frac{1}{2}+\frac{2}{p}}}^{p \alpha(r)} \\
& \times\left(\left\|v^{3}\left(t^{\prime}\right)\right\|_{\dot{H}^{\frac{1}{2}+\frac{2}{p}}}^{p}\left\|\omega_{\frac{r}{2}}\left(t^{\prime}\right)\right\|_{L^{2}}^{2}\right)^{\frac{1}{p}-\alpha(r)}\left\|\nabla \omega_{\frac{r}{2}}\left(t^{\prime}\right)\right\|_{L^{2}}^{2\left(\frac{1}{2}-\frac{1}{p}\right)} d t^{\prime} .
\end{aligned}
$$

As we have

$$
\frac{1}{2}+\alpha(r)+\left(\frac{1}{p}-\alpha(r)\right)+\left(\frac{1}{2}-\frac{1}{p}\right)=1,
$$

applying Hölder inequality ensures that

$$
\begin{aligned}
\left|F_{3}(t)\right| \lesssim\left(\int_{0}^{t}\left\|\partial_{3}^{2} v^{3}\left(t^{\prime}\right)\right\|_{\mathcal{H}^{\theta, r}}^{2} d t^{\prime}\right)^{\frac{1}{2}}\left(\int_{0}^{t}\left\|v^{3}\left(t^{\prime}\right)\right\|_{\dot{H}^{\frac{1}{2}+\frac{2}{p}}}^{p} d t^{\prime}\right)^{\alpha(r)} \\
\times\left(\int_{0}^{t}\left\|v^{3}\left(t^{\prime}\right)\right\|_{\dot{H}^{\frac{1}{2} ;+\frac{2}{p}}}^{p}\left\|\omega_{\frac{r}{2}}\left(t^{\prime}\right)\right\|_{L^{2}}^{2} d t^{\prime}\right)^{\frac{1}{p}-\alpha(r)}\left(\int_{0}^{t}\left\|\nabla \omega_{\frac{r}{2}}\left(t^{\prime}\right)\right\|_{L^{2}}^{2} d t^{\prime}\right)^{\frac{1}{2}-\frac{1}{p}} .
\end{aligned}
$$

Applying the convexity inequality leads to

$$
\begin{aligned}
\left|F_{3}(t)\right| \leq \frac{r-1}{r^{2}} \int_{0}^{t}\left\|\nabla \omega_{\frac{r}{2}}\left(t^{\prime}\right)\right\|_{L^{2}}^{2} d t^{\prime}+C \int_{0}^{t}\left\|v^{3}\left(t^{\prime}\right)\right\|_{\dot{H}^{\frac{1}{2}+\frac{2}{p}}}^{p}\left\|\omega_{\frac{r}{2}}\left(t^{\prime}\right)\right\|_{L^{2}}^{2} d t^{\prime} \\
+C\left(\int_{0}^{t}\left\|v^{3}\left(t^{\prime}\right)\right\|_{\dot{H}^{\frac{1}{2}+\frac{2}{p}}}^{p} d t^{\prime}\right)^{1-\frac{r}{2}}\left(\int_{0}^{t}\left\|\partial_{3}^{2} v^{3}\left(t^{\prime}\right)\right\|_{\mathcal{H}^{\theta, r}}^{2} d t^{\prime}\right)^{\frac{r}{2}} .
\end{aligned}
$$

Conclusion of the proof to Proposition 2.1. Resuming the Estimates (4.2), (4.10) and (4.11) into (4.1), we obtain

$$
\frac{1}{r}\left\|\omega_{\frac{r}{2}}(t)\right\|_{L^{2}}^{2}+\frac{r-1}{r^{2}} \int_{0}^{t}\left\|\nabla \omega_{\frac{r}{2}}\left(t^{\prime}\right)\right\|_{L^{2}}^{2} d t^{\prime}
$$

$$
+C \int_{0}^{t}\left\|v^{3}\left(t^{\prime}\right)\right\|_{\dot{H}^{\frac{1}{2}+\frac{2}{p}}}^{p}\left\|\omega_{\frac{r}{2}}\left(t^{\prime}\right)\right\|_{L^{2}}^{2} d t^{\prime}
$$

Inequality (2.8) follows from Gronwall lemma once notice that $x^{\frac{1}{4}} e^{C x} \lesssim e^{C^{\prime} x}$ for $C^{\prime}>C$.

5. Proof of the estimate for the Second Vertical Derivatives of $v^{3}$

In this section, we shall present the proof of Proposition 2.2. Let $\mathcal{H}^{\theta, r}$ be given by Definition 2.1. We get, by taking the $\mathcal{H}^{\theta, r}$ inner product of the $\partial_{3} v^{3}$ equation of $(\widetilde{N S})$ with $\partial_{3} v^{3}$, 
that

$$
\begin{aligned}
\frac{1}{2} \frac{d}{d t}\left\|\partial_{3} v^{3}(t)\right\|_{\mathcal{H}^{\theta, r}}^{2}+\left\|\nabla \partial_{3} v^{3}(t)\right\|_{\mathcal{H}^{\theta, r}}^{2}=-\sum_{n=1}^{3}\left(Q_{n}(v, v) \mid \partial_{3} v^{3}\right)_{\mathcal{H}^{\theta, r}} \quad \text { with } \\
Q_{1}(v, v) \stackrel{\text { def }}{=}\left(\operatorname{Id}+\partial_{3}^{2} \Delta^{-1}\right)\left(\partial_{3} v^{3}\right)^{2}+\partial_{3}^{2} \Delta^{-1}\left(\sum_{\ell, m=1}^{2} \partial_{\ell} v^{m} \partial_{m} v^{\ell}\right), \\
Q_{2}(v, v) \stackrel{\text { def }}{=}\left(\operatorname{Id}+2 \partial_{3}^{2} \Delta^{-1}\right)\left(\sum_{\ell=1}^{2} \partial_{3} v^{\ell} \partial_{\ell} v^{3}\right) \text { and } \\
Q_{3}(v, v) \stackrel{\text { def }}{=} v \cdot \nabla \partial_{3} v^{3} .
\end{aligned}
$$

The estimate involving $Q_{1}$ relies on the following lemma. Let us point out that this term $Q_{1}$ contains terms which are quadratic with respect to $v_{\text {curl }}^{\mathrm{h}}$.

Lemma 5.1. Let $A$ be a bounded Fourier multiplier. If $p$ and $\theta$ satisfy

$$
0<\theta<\frac{1}{2}-\frac{1}{p}
$$

then we have

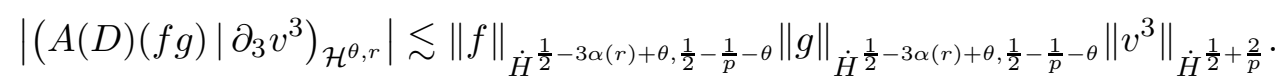

Proof. Let us first observe that, for any couple $(\alpha, \beta)$ in $\mathbb{R}^{2}$, we have, thanks to CauchySchwartz inequality, that, for any real valued function $a$ and $b$,

$$
\begin{aligned}
\left|(a \mid b)_{\mathcal{H}^{\theta, r}}\right| & =\left.\left|\int_{\mathbb{R}^{3}}\right| \xi_{\mathrm{h}}\right|^{-6 \alpha(r)+2 \theta-\alpha}\left|\xi_{3}\right|^{-\beta-2 \theta} \widehat{a}(\xi)\left|\xi_{\mathrm{h}}\right|^{\alpha}\left|\xi_{3}\right|^{\beta \widehat{b}}(-\xi) d \xi \mid \\
& \leq\|a\|_{\dot{H}^{-6 \alpha(r)+2 \theta-\alpha,-\beta-2 \theta}}\|b\|_{\dot{H}^{\alpha, \beta}} .
\end{aligned}
$$

As $A(D)$ is a bounded Fourier multiplier, applying (5.3) with $\alpha=0$ and $\beta=-\frac{1}{2}+\frac{2}{p}$, we obtain

$$
\left|\left(A(D)(f g) \mid \partial_{3} v^{3}\right)_{\mathcal{H}^{\theta, r}}\right| \lesssim\|f g\|_{\dot{H}^{-6 \alpha(r)+2 \theta, \frac{1}{2}-\frac{2}{p}-2 \theta}}\left\|\partial_{3} v^{3}\right\|_{\dot{H}^{0,-\frac{1}{2}+\frac{2}{p}}} .
$$

Because $\dot{H}^{s, s^{\prime}}=\left(\dot{B}_{2,2}^{s}\right)_{\mathrm{h}}\left(\dot{B}_{2,2}^{s^{\prime}}\right)_{\mathrm{v}}, r$ is in $] 3 / 2,2\left[, 3 \alpha(r)\right.$ is less than $\frac{1}{2} \mathrm{i} \mathrm{BR}_{\dot{\mathrm{g}}}$ and thanks to Condition (5.2), law of products of Lemma 3.7 implies in particular that

$$
\|f g\|_{\dot{H}^{-6 \alpha(r)+2 \theta, \frac{1}{2}-\frac{2}{p}-2 \theta}} \lesssim\|f\|_{\dot{H}^{\frac{1}{2}-3 \alpha(r)+\theta, \frac{1}{2}-\frac{1}{p}-\theta}}\|g\|_{\dot{H}^{\frac{1}{2}-3 \alpha(r)+\theta, \frac{1}{2}-\frac{1}{p}-\theta}} .
$$

Due to Lemma 3.4, we have

$$
\left\|\partial_{3} v^{3}\right\|_{\dot{H}^{0,-\frac{1}{2}+\frac{2}{p}}} \lesssim\left\|v^{3}\right\|_{\dot{H}^{0, \frac{1}{2}+\frac{2}{p}}} \leq\left\|v^{3}\right\|_{\dot{H}^{\frac{1}{2}+\frac{2}{p}}}
$$

and thus the lemma is proved.

Because both $\partial_{3}^{2} \Delta^{-1}$ and $\partial_{\mathrm{h}}^{2} \Delta_{\mathrm{h}}^{-1}$ are bounded Fourier multipliers, applying Lemma 5.1 with $f$ and $g$ of the form $\partial_{\mathrm{h}} v_{\text {curl }}^{\mathrm{h}}$ or $\partial_{\mathrm{h}} v_{\text {div }}^{\mathrm{h}}$ or with $f=g=\partial_{3} v^{3}$ gives,

$$
\left|\left(Q_{1}(v, v) \mid \partial_{3} v^{3}\right)_{\mathcal{H}^{\theta, r}}\right| \lesssim\left\|v^{3}\right\|_{\dot{H}^{\frac{1}{2}+\frac{2}{p}}}\left(\|\omega\|_{\dot{H}^{\frac{1}{2}-3 \alpha(r)+\theta, \frac{1}{2}-\frac{1}{p}-\theta}}^{2}+\left\|\partial_{3} v^{3}\right\|_{\dot{H}^{\frac{1}{2}-3 \alpha(r)+\theta, \frac{1}{2}-\frac{1}{p}-\theta}}^{2}\right) .
$$


Because $p>4, r>4 / 3$, we have $\frac{1}{p}+\frac{1}{r}<1$, and $\theta<\alpha(r)<1 / 2-1 / p$ so that the Condition (5.2) is satisfied. Then we get, by using Lemma 3.5 and Lemma 3.1, that

$$
\|\omega\|_{\dot{H}^{\frac{1}{2}-3 \alpha(r)+\theta, \frac{1}{2}-\frac{1}{p}-\theta}} \leq\|\omega\|_{\dot{H}^{1-3 \alpha(r)-\frac{1}{p}}} \lesssim\left\|\omega_{\frac{r}{2}}\right\|_{L^{2}}^{2 \alpha(r)+\frac{1}{p}}\left\|\nabla \omega_{\frac{r}{2}}\right\|_{L^{2}}^{\frac{1}{p^{\prime}}} .
$$

While it follows from Definition 2.1 that

$$
\begin{aligned}
\|a\|_{\dot{H}^{\frac{1}{2}-3 \alpha(r)+\theta, \frac{1}{2}-\frac{1}{p}-\theta}}^{2} & =\int_{\mathbb{R}^{3}}\left|\xi_{\mathrm{h}}\right|^{1-6 \alpha(r)+2 \theta}\left|\xi_{3}\right|^{1-\frac{2}{p}-2 \theta}|\widehat{a}(\xi)|^{2} d \xi \\
& \leq \int_{\mathbb{R}^{3}}|\widehat{a}(\xi)|^{\frac{2}{p}}(|\xi||\widehat{a}(\xi)|)^{\frac{2}{p^{\prime}}}\left|\xi_{\mathrm{h}}\right|^{2(-3 \alpha(r)+\theta)}\left|\xi_{3}\right|^{-2 \theta} d \xi .
\end{aligned}
$$

Applying Hölder's inequality with measure $\left|\xi_{\mathrm{h}}\right|^{2(-3 \alpha(r)+\theta)}\left|\xi_{3}\right|^{-2 \theta} d \xi$ yields

$$
\|a\|_{\dot{H}^{\frac{1}{2}-3 \alpha(r)+\theta, \frac{1}{2} n b s p ;-\frac{1}{p}-\theta}} \leq\|a\|_{\mathcal{H}^{\theta, r}}^{\frac{1}{p}}\|\nabla a\|_{\mathcal{H}^{\theta, r}}^{\frac{1}{p^{\prime}}} .
$$

We then infer that

$$
\left|\left(Q_{1}(v, v) \mid \partial_{3} v^{3}\right)_{\mathcal{H}^{\theta, r}}\right| \lesssim\left\|v^{3}\right\|_{\dot{H}^{\frac{1}{2}+\frac{2}{p}}}\left(\left\|\omega_{\frac{r}{2}}\right\|_{L^{2}}^{2\left(\frac{1}{p}+2 \alpha(r)\right)}\left\|\nabla \omega_{\frac{r}{2}}\right\|_{L^{2}}^{\frac{2}{p^{\prime}}}+\left\|\partial_{3} v^{3}\right\|_{\mathcal{H}^{\theta, r}}^{\frac{2}{p}}\left\|\nabla \partial_{3} v^{3}\right\|_{\mathcal{H}^{\theta, r}}^{\frac{2}{p^{\prime}}}\right) .
$$

Convexity inequality ensures

$$
\begin{aligned}
\left|\left(Q_{1}(v, v) \mid \partial_{3} v^{3}\right)_{\mathcal{H}^{\theta, r}}\right| \leq \frac{1}{6}\left\|\nabla \partial_{3} v^{3}\right\|_{\mathcal{H}^{\theta, r}}^{2}+C\left\|v^{3}\right\|_{\dot{H}^{\frac{1}{2}+\frac{2}{p}}}^{p}\left\|\partial_{3} v^{3}\right\|_{\mathcal{H}^{\theta, r}}^{2} & \\
& +C\left\|v^{3}\right\|_{\dot{H}^{\frac{1}{2}+\frac{2}{p}}}\left\|\omega_{\frac{r}{2}}\right\|_{L^{2}}^{2\left(\frac{1}{p}+2 \alpha(r)\right)}\left\|\nabla \omega_{\frac{r}{2}}\right\|_{L^{2}}^{\frac{2}{p^{\prime}}}
\end{aligned}
$$

In order to estimate $\left(Q_{2}(v, v) \mid \partial_{3} v^{3}\right)_{\mathcal{H}^{\theta, r}}$, we first make the following observation: since $\theta>3 \alpha(r)-2 / p$ and $4<p<\frac{2 r}{2-r}$, we have

$$
\frac{2}{p}+3 \alpha(r)-1 \leq \theta<5 \alpha(r)<\frac{2}{p}+3 \alpha(r),
$$

and hence

$$
\left|\xi_{\mathrm{h}}\right|^{2\left(1-\frac{2}{p}-6 \alpha(r)+2 \theta\right)}\left|\xi_{3}\right|^{2\left(\frac{2}{p}+3 \alpha(r)-2 \theta\right)} \leq\left|\xi_{\mathrm{h}}\right|^{2(-3 \alpha(r)+\theta)}\left|\xi_{3}\right|^{-2 \theta}|\xi|^{2} .
$$

We infer that

$$
\begin{aligned}
\|a\|_{\dot{H}^{1-6 \alpha(r)-\frac{2}{p}+2 \theta, \frac{2}{p}+3 \alpha(r)-2 \theta}}^{2} & =\int_{\mathbb{R}^{3}}\left|\xi_{\mathrm{h}}\right|^{2\left(1-\frac{2}{p}-6 \alpha(r)+2 \theta\right)}\left|\xi_{3}\right|^{2\left(\frac{2}{p}+3 \alpha(r)-2 \theta\right)}|\widehat{a}(\xi)|^{2} d \xi \\
& \leq \int_{\mathbb{R}^{3}}\left|\xi_{\mathrm{h}}\right|^{2(-3 \alpha(r)+\theta)}\left|\xi_{3}\right|^{-2 \theta}(|\xi \| \widehat{a}(\xi)|)^{2} d \xi \\
& =\|\nabla a\|_{\mathcal{H}^{\theta, r}}^{2} .
\end{aligned}
$$

Along the same lines, one has

$$
\|a\|_{\dot{H}^{1-6 \alpha(r)+2 \theta, 3 \alpha(r)-2 \theta}}^{2} \leq\|\nabla a\|_{\mathcal{H}^{\theta, r}}^{2} .
$$

While we get by applying Bony's decomposition (3.12) in the vertical variable for $\partial_{3} v^{\ell} \partial_{\ell} v^{3}$ that

$$
\partial_{3} v^{\ell} \partial_{\ell} v^{3}=T^{\mathrm{v}}\left(\partial_{3} v^{\ell}, \partial_{\ell} v^{3}\right)+\bar{T}^{\mathrm{v}}\left(\partial_{3} v^{\ell}, \partial_{\ell} v^{3}\right)+R^{\mathrm{v}}\left(\partial_{3} v^{\ell}, \partial_{\ell} v^{3}\right) .
$$


The law of product of Lemma 3.7 implies that

$$
\begin{aligned}
& \left\|T^{\mathrm{v}}\left(\partial_{3} v^{\ell}, \partial_{\ell} v^{3}\right)+\bar{T}^{\mathrm{v}}\left(\partial_{3} v^{\ell}, \partial_{\ell} v^{3}\right)\right\|_{\dot{H}^{\frac{2}{p}-1,-3 \alpha(r)-\frac{2}{p}}}
\end{aligned}
$$

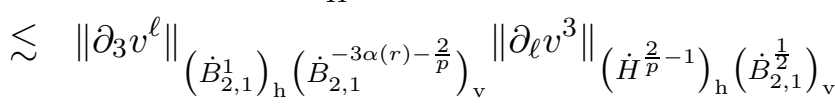

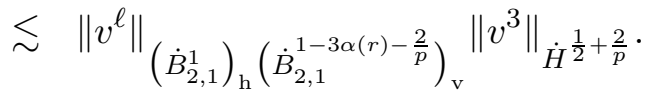

As we have $\left\|\partial_{\ell} v^{3}\right\|_{\left(\dot{H}^{\frac{2}{p}-1}\right)_{\mathrm{h}}\left(\dot{B}_{2,1}^{\frac{1}{2}}\right)_{\mathrm{v}}} \lesssim\left\|v^{3}\right\|_{\left(\dot{H}^{\frac{2}{p}}\right)_{\mathrm{h}}\left(\dot{B}_{2,1}^{\frac{1}{2}}\right)_{\mathrm{v}}} \leq\left\|v^{3}\right\|_{\dot{H}^{\frac{1}{2}+\frac{2}{p}}}$.

Thus we get, by applying (5.3) that

$$
\begin{aligned}
\mid((\mathrm{Id} & \left.\left.+2 \partial_{3}^{2} \Delta^{-1}\right) \sum_{\ell=1}^{2}\left(T^{\mathrm{v}}\left(\partial_{3} v^{\ell}, \partial_{\ell} v^{3}\right)+\bar{T}^{\mathrm{v}}\left(\partial_{3} v^{\ell}, \partial_{\ell} v^{3}\right)\right) \mid \partial_{3} v^{3}\right)_{\mathcal{H}^{\theta, r}} \mid \\
& \lesssim\left\|T^{\mathrm{v}}\left(\partial_{3} v^{\ell}, \partial_{\ell} v^{3}\right)+\bar{T}^{\mathrm{v}}\left(\partial_{3} v^{\ell}, \partial_{\ell} v^{3}\right)\right\|_{\dot{H}^{\frac{2}{p}-1,-3 \alpha(r)-\frac{2}{p}}}\left\|\partial_{3} v^{3}\right\|_{\dot{H}^{1-6 \alpha(r)-\frac{2}{p}+2 \theta, \frac{2}{p}+3 \alpha(r)-2 \theta}} \\
& \lesssim\left\|v^{3}\right\|_{\dot{H}^{\frac{1}{2}+\frac{2}{p}}}\left\|v^{\mathrm{h}}\right\|_{\left(\dot{B}_{2,1}^{1}\right)_{\mathrm{h}}\left(\dot{B}_{2,1}^{1-3 \alpha(r)-\frac{2}{p}}\right)_{\mathrm{v}}\left\|\nabla \partial_{3} v^{3}\right\|_{\mathcal{H}^{\theta, r}} .}
\end{aligned}
$$

Whereas applying the law of product of Lemma 3.7 once again yields

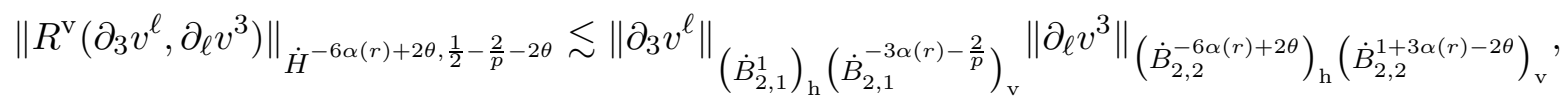

which together with (5.4) and (5.9) ensures

$$
\begin{aligned}
\mid\left(\left(\operatorname{Id}+2 \partial_{3}^{2} \Delta^{-1}\right)\right. & \left.\sum_{\ell=1}^{2} R^{\mathrm{v}}\left(\partial_{3} v^{\ell}, \partial_{\ell} v^{3}\right) \mid \partial_{3} v^{3}\right)_{\mathcal{H}^{\theta, r}} \mid \\
& \lesssim\left\|R^{\mathrm{v}}\left(\partial_{3} v^{\ell}, \partial_{\ell} v^{3}\right)\right\|_{\dot{H}^{-6 \alpha(r)+2 \theta, \frac{1}{2}-\frac{2}{p}-2 \theta}}\left\|\partial_{3} v^{3}\right\|_{\dot{H}^{0,-\frac{1}{2}+\frac{2}{p}}} \\
& \lesssim\left\|v^{3}\right\|_{\dot{H}^{\frac{1}{2}+\frac{2}{p}}}\left\|v^{\mathrm{h}}\right\|_{\left(\dot{B}_{2,1}^{1}\right)_{\mathrm{h}}\left(\dot{B}_{2,1}^{1-3 \alpha(r)-\frac{2}{p}}\right)_{\mathrm{v}}\left\|\nabla \partial_{3} v^{3}\right\|_{\mathcal{H}^{\theta, r}}}
\end{aligned}
$$

Therefore, by virtue of Proposition 3.1, we infer that

$$
\begin{aligned}
\left|\left(Q_{2}(v, v) \mid \partial_{3} v^{3}\right)_{\mathcal{H}^{\theta, r}}\right| \lesssim & \left\|v^{3}\right\|_{\dot{H}^{\frac{1}{2}+\frac{2}{p}}} \\
& \times\left(\left\|\omega_{\frac{r}{2}}\right\|_{L^{2}}^{2\left(\alpha(r)+\frac{1}{p}\right)}\left\|\nabla \omega_{\frac{r}{2}}\right\|_{L^{2}}^{1-\frac{2}{p}}+\left\|\partial_{3} v^{3}\right\|_{\mathcal{H}^{\theta, r}}^{\frac{2}{p}}\left\|\nabla \partial_{3} v^{3}\right\|_{\mathcal{H}^{\theta, r}}^{1-\frac{2}{p}}\right)\left\|\nabla \partial_{3} v^{3}\right\|_{\mathcal{H}^{\theta, r} .} .
\end{aligned}
$$

Applying convexity inequality yields

$$
\begin{aligned}
\left|\left(Q_{2}(v, v) \mid \partial_{3} v^{3}\right)_{\mathcal{H}^{\theta, r}}\right| \leq \frac{1}{6}\left\|\nabla \partial_{3} v^{3}\right\|_{\mathcal{H}^{\theta, r}}^{2}+C\left\|v^{3}\right\|_{\dot{H}^{\frac{1}{2}+\frac{2}{p}}}^{p}\left\|\partial_{3} v^{3}\right\|_{\mathcal{H}^{\theta, r}}^{2} & \\
& +C\left\|v^{3}\right\|_{\dot{H}^{\frac{1}{2}+\frac{2}{p}}}^{2}\left\|\omega_{\frac{r}{2}}\right\|_{L^{2}}^{4\left(\alpha(r)+\frac{1}{p}\right)}\left\|\nabla \omega_{\frac{3}{4}}\right\|_{L^{2}}^{2\left(1-\frac{2}{p}\right)} .
\end{aligned}
$$

Finally let us estimate $\left(Q_{3}(v, v) \mid \partial_{3} v^{3}\right)_{\mathcal{H}^{\theta, r}}$.

Lemma 5.2. We have the following inequality.

$$
\begin{aligned}
\left|\left(v^{\mathrm{h}} \cdot \nabla_{\mathrm{h}} \partial_{3} v^{3} \mid \partial_{3} v^{3}\right)_{\mathcal{H}^{\theta, r}}\right| \lesssim\left\|v^{3}\right\|_{\dot{H}^{\frac{1}{2}+\frac{2}{p}}}\left(\left\|\nabla_{\mathrm{h}} v^{\mathrm{h}}\right\|_{\dot{H}^{\frac{1}{2}-3 \alpha(r)+\theta, \frac{1}{2}-\frac{1}{p}-\theta}}^{2}\right. \\
\quad+\left\|\partial_{3} v^{3}\right\|_{\dot{H}^{\frac{1}{2}-3 \alpha(r)+\theta, \frac{1}{2}-\frac{1}{p}-\theta}}^{2}+\left\|v^{\mathrm{h}}\right\|_{\left.\left(\dot{B}_{2,1}^{1}\right)_{\mathrm{h}}\left(\dot{B}_{2,1}^{1-3 \alpha(r)-\frac{2}{p}}\right)_{\mathrm{v}}\left\|\nabla \partial_{3} v^{3}\right\|_{\mathcal{H}^{\theta, r}}\right) .} .
\end{aligned}
$$


Proof. Let us use the following alternative definition for the inner-product in $\mathcal{H}^{\theta, r}$ : based on the fact that the space $\mathcal{H}^{\theta, r}$ is equal to the space $\left(\dot{B}_{2,2}^{-3 \alpha(r)+\theta}\right)_{\mathrm{h}}\left(\dot{B}_{2,2}^{-\theta}\right)_{\mathrm{v}}$ of De finition 3.2 .

$$
\left(v^{\mathrm{h}} \cdot \nabla_{\mathrm{h}} \partial_{3} v^{3} \mid \partial_{3} v^{3}\right)_{\mathcal{H}^{\theta, r}}=\sum_{k, \ell \in \mathbb{Z}^{2}} 2^{2 k(-3 \alpha(r)+\theta)} 2^{-2 \ell \theta}\left(\Delta_{k}^{\mathrm{h}} \Delta_{\ell}^{\mathrm{v}}\left(v^{\mathrm{h}} \cdot \nabla_{\mathrm{h}} \partial_{3} v^{3}\right) \mid \Delta_{k}^{\mathrm{h}} \Delta_{\ell}^{\mathrm{v}} \partial_{3} v^{3}\right)_{L^{2}} .
$$

By using Bony's decomposition (3.12) to $v^{\mathrm{h}} \cdot \nabla_{\mathrm{h}} \partial_{3} v^{3}$ for both horizontal and vertical variables, we write that

$$
\begin{aligned}
v^{\mathrm{h}} \cdot \nabla_{\mathrm{h}} \partial_{3} v^{3}= & \left(T^{\mathrm{h}}+R^{\mathrm{h}}+\bar{T}^{\mathrm{h}}\right)\left(T^{\mathrm{v}}+R^{\mathrm{v}}+\bar{T}^{\mathrm{v}}\right)\left(v^{\mathrm{h}}, \nabla_{\mathrm{h}} \partial_{3} v^{3}\right) \\
= & T^{\mathrm{h}} T^{\mathrm{v}}\left(v^{\mathrm{h}}, \nabla_{\mathrm{h}} \partial_{3} v^{3}\right)+A+B \quad \text { with } \\
A \stackrel{\text { def }}{=} & T^{\mathrm{h}} R^{\mathrm{v}}\left(v^{\mathrm{h}}, \nabla_{\mathrm{h}} \partial_{3} v^{3}\right)+T^{\mathrm{h}} \bar{T}^{\mathrm{v}}\left(v^{\mathrm{h}}, \nabla_{\mathrm{h}} \partial_{3} v^{3}\right) \\
B \stackrel{\text { def }}{=} & R^{\mathrm{h}} T^{\mathrm{v}}\left(v^{\mathrm{h}}, \nabla_{\mathrm{h}} \partial_{3} v^{3}\right)+R^{\mathrm{h}} R^{\mathrm{v}}\left(v^{\mathrm{h}}, \nabla_{\mathrm{h}} \partial_{3} v^{3}\right)+R^{\mathrm{h}} \bar{T}^{\mathrm{v}}\left(v^{\mathrm{h}}, \nabla_{\mathrm{h}} \partial_{3} v^{3}\right) \\
& \quad+\bar{T}^{\mathrm{h}} T^{\mathrm{v}}\left(v^{\mathrm{h}}, \nabla_{\mathrm{h}} \partial_{3} v^{3}\right)+\bar{T}^{\mathrm{h}} R^{\mathrm{v}}\left(v^{\mathrm{h}}, \nabla_{\mathrm{h}} \partial_{3} v^{3}\right)+\bar{T}^{\mathrm{h}} \bar{T}^{\mathrm{v}}\left(v^{\mathrm{h}}, \nabla_{\mathrm{h}} \partial_{3} v^{3}\right) .
\end{aligned}
$$

- The estimate of $\left(\Delta_{k}^{\mathrm{h}} \Delta_{\ell}^{\mathrm{v}} T^{\mathrm{h}} T^{\mathrm{v}}\left(v^{\mathrm{h}}, \nabla_{\mathrm{h}} \partial_{3} v^{3}\right) \mid \Delta_{k}^{\mathrm{h}} \Delta_{\ell}^{\mathrm{v}} \partial_{3} v^{3}\right)_{L^{2}}$.

By applying commutator's argument and also considering the support to the Fourier transform of the terms in $T^{\mathrm{h}} T^{\mathrm{v}}\left(v^{\mathrm{h}}, \nabla_{\mathrm{h}} \partial_{3} v^{3}\right)$, we write

$$
\begin{aligned}
\left(\Delta_{k}^{\mathrm{h}} \Delta_{\ell}^{\mathrm{v}} T^{\mathrm{h}} T^{\mathrm{v}}\left(v^{\mathrm{h}}, \nabla_{\mathrm{h}} \partial_{3} v^{3}\right) \mid \Delta_{k}^{\mathrm{h}} \Delta_{\ell}^{\mathrm{v}} \partial_{3} v^{3}\right)_{L^{2}} \stackrel{\text { def }}{=} I_{k, \ell}^{1}+I_{k, \ell}^{2}+I_{k, \ell}^{3} \quad \text { with } \\
I_{k, \ell}^{1} \stackrel{\text { def }}{=} \sum_{\left|k^{\prime}-k\right| \leq 4}\left(\left[\Delta_{k}^{\mathrm{h}} \Delta_{\ell}^{\mathrm{v}}, S_{k^{\prime}-1}^{\mathrm{h}} S_{\ell^{\prime}-1}^{\mathrm{v}} v^{\mathrm{h}}\right] \Delta_{k^{\prime}}^{\mathrm{h}} \Delta_{\ell^{\prime}}^{\mathrm{v}} \nabla_{\mathrm{h}} \partial_{3} v^{3} \mid \Delta_{k}^{\mathrm{h}} \Delta_{\ell}^{\mathrm{v}} \partial_{3} v^{3}\right)_{L^{2}}, \\
I_{k, \ell}^{2} \stackrel{\text { def }}{=} \sum_{\left|\ell^{\prime}-\ell\right| \leq 4}\left(\left(S_{k^{\prime}-1}^{\mathrm{h}} S_{\ell^{\prime}-1}^{\mathrm{v}} v^{\mathrm{h}}-S_{k-1}^{\mathrm{h}} S_{\ell-1}^{\mathrm{v}} v^{\mathrm{h}}\right) \Delta_{k^{\prime}}^{\mathrm{h}} \Delta_{\ell^{\prime}}^{\mathrm{v}} \Delta_{k}^{\mathrm{h}} \Delta_{\ell}^{\mathrm{v}} n a_{\mathrm{h}} \partial_{3} v^{3} \mid \Delta_{k}^{\mathrm{h}} \Delta_{\ell}^{\mathrm{v}} \partial_{3} v^{3}\right)_{L^{2}} \\
I_{k, \ell}^{3} \stackrel{\text { def }}{=}-\frac{1}{2}\left(S_{k-1}^{\mathrm{h}} S_{\ell-1}^{\mathrm{v}} \operatorname{div}_{\mathrm{h}} v^{\mathrm{h}} \Delta_{k}^{\mathrm{h}} \Delta_{\ell}^{\mathrm{v}} \partial_{3} v^{3} \mid \Delta_{k}^{\mathrm{h}} \Delta_{\ell}^{\mathrm{v}} \partial_{3} v^{3}\right)_{L^{2}} .
\end{aligned}
$$

and

It follows from a standard commutator's estimate (see for instance [1]) that

$$
\begin{aligned}
&\left|I_{k, \ell}^{1}\right| \lesssim \sum_{\substack{\left|k^{\prime}-k\right| \leq 4 \\
\left|\ell^{\prime}-\ell\right| \leq 4}}\left(2^{-k}\left\|S_{k^{\prime}-1}^{\mathrm{h}} S_{\ell^{\prime}-1}^{\mathrm{v}} \nabla_{\mathrm{h}} v^{\mathrm{h}}\right\|_{L^{\infty}}\right. \\
&\left.\quad+2^{-\ell}\left\|S_{k^{\prime}-1}^{\mathrm{h}} S_{\ell^{\prime}-1}^{\mathrm{v}} \partial_{3} v^{\mathrm{h}}\right\|_{L^{\infty}}\right)\left\|\Delta_{k^{\prime}}^{\mathrm{h}} \Delta_{\ell^{\prime}}^{\mathrm{v}} \nabla_{\mathrm{h}} \partial_{3} v^{3}\right\|_{L^{2}}\left\|\Delta_{k}^{\mathrm{h}} \Delta_{\ell}^{\mathrm{v}} \partial_{3} v^{3}\right\|_{L^{2}}
\end{aligned}
$$

Note that applying Lemma 3.3 gives

$$
\left\|S_{k^{\prime}-1}^{\mathrm{h}} S_{\ell^{\prime}-1}^{\mathrm{v}} \nabla_{\mathrm{h}} v^{\mathrm{h}}\right\|_{L^{\infty}} \lesssim 2^{k^{\prime}\left(\frac{1}{2}+3 \alpha(r)-\theta\right)} 2^{\ell^{\prime}\left(\frac{1}{p}+\theta\right)}\left\|\nabla_{\mathrm{h}} v^{\mathrm{h}}\right\|_{\dot{H}^{\frac{1}{2}-3 \alpha(r)+\theta, \frac{1}{2}-\frac{1}{p}-\theta}},
$$


from which, we infer that

$$
\begin{aligned}
& 2^{-k} \sum_{\substack{\left|k^{\prime}-k\right| \leq 4 \\
\left|\ell^{\prime}-\ell\right| \leq 4}}\left\|S_{k^{\prime}-1}^{\mathrm{h}} S_{\ell^{\prime}-1}^{\mathrm{v}} \nabla_{\mathrm{h}} v^{\mathrm{h}}\right\|_{L^{\infty}}\left\|\Delta_{k^{\prime}}^{\mathrm{h}} \Delta_{\ell^{\prime}}^{\mathrm{v}} \nabla_{\mathrm{h}} \partial_{3} v^{3}\right\|_{L^{2}}\left\|\Delta_{k}^{\mathrm{h}} \Delta_{\ell}^{\mathrm{v}} \partial_{3} v^{3}\right\|_{L^{2}} \\
& \vdots \sum_{\substack{\left|k^{\prime}-k\right| \leq 4 \\
\left|\ell^{\prime}-\ell\right| \leq 4}} c_{k^{\prime}, \ell^{\prime}} 2^{2 k^{\prime}(3 \alpha(r)-\theta)} 2^{\ell^{\prime}\left(-\frac{1}{2}+\frac{2}{p}+2 \theta\right)}\left\|\nabla_{\mathrm{h}} v^{\mathrm{h}}\right\|_{\dot{H}^{\frac{1}{2}-3 \alpha(r)+\theta, \frac{1}{2}-\frac{1}{p}-\theta}} \\
& \times\left\|\partial_{3} v^{3}\right\|_{\dot{H}^{\frac{1}{2}-3 \alpha(r)+\theta, \frac{1}{2}-\frac{1}{p}-\theta} c_{k, \ell} 2^{\ell\left(\frac{1}{2}-\frac{2}{p}\right)}\left\|\partial_{3} v^{3}\right\|_{\dot{H}^{0,-\frac{1}{2}+\frac{2}{p}}}} \\
& \lesssim d_{k, \ell} 2^{2 k(3 \alpha(r)-\theta)} 2^{2 \ell \theta}\left\|v^{3}\right\|_{\dot{H}^{\frac{1}{2}+\frac{2}{p}}}\left(\left\|\nabla_{\mathrm{h}} v^{\mathrm{h}}\right\|_{\dot{H}^{\frac{1}{2}-3 \alpha(r)+\theta, \frac{1}{2}-\frac{1}{p}-\theta}}^{2}+\left\|\partial_{3} v^{3}\right\|_{\dot{H}^{\frac{1}{2}-3 \alpha(r)+\theta, \frac{1}{2}-\frac{1}{p}-\theta}}^{2}\right) .
\end{aligned}
$$

Here and in what follows, we always denote $\left(c_{k, \ell}\right)_{k, \ell \in \mathbb{Z}^{2}}$ (resp. $\left(d_{k, \ell}\right)_{k, \ell \in \mathbb{Z}^{2}}$ to be a generic element of the sphere in $\ell^{2}\left(\mathbb{Z}^{2}\right)$ (resp. $\ell^{1}\left(\mathbb{Z}^{2}\right)$ ). The same estimate holds for $I_{k, \ell}^{3}$.

Likewise, since

$$
\left\|S_{k^{\prime}-1}^{\mathrm{h}} S_{\ell^{\prime}-1}^{\mathrm{v}} \partial_{3} v^{\mathrm{h}}\right\|_{L^{\infty}} \lesssim 2^{\ell^{\prime}\left(\frac{1}{2}+3 \alpha(r)+\frac{2}{p}\right)}\left\|v^{\mathrm{h}}\right\|_{\left(\dot{B}_{2,1}^{1}\right)_{\mathrm{h}}\left(\dot{B}_{2,1}^{1-3 \alpha(r)-\frac{2}{p}}\right)_{\mathrm{v}}},
$$

and $\left\|\nabla_{\mathrm{h}} v^{3}\right\|_{\left(\dot{H}^{-1+\frac{2}{p}}\right)_{\mathrm{h}}\left(\dot{B}_{2,1}^{\frac{1}{2}}\right)_{\mathrm{v}}} \lesssim\left\|v^{3}\right\|_{\left(\dot{H}^{\frac{2}{p}}\right)_{\mathrm{h}}\left(\dot{B}_{2,1}^{\frac{1}{2}}\right)_{\mathrm{v}}} \lesssim\left\|v^{3}\right\|_{\dot{H}^{\frac{1}{2}+\frac{2}{p}}}$, we have

$$
\begin{aligned}
& 2^{-\ell} \sum_{\substack{\left|k^{\prime}-k\right| \leq 4 \\
\left|\ell^{\prime}-\ell\right| \leq 4}}\left\|S_{k^{\prime}-1}^{\mathrm{h}} S_{\ell^{\prime}-1}^{\mathrm{v}} \partial_{3} v^{\mathrm{h}}\right\|_{L^{\infty}}\left\|\Delta_{k^{\prime}}^{\mathrm{h}} \Delta_{\ell^{\prime}}^{\mathrm{v}} \nabla_{\mathrm{h}} \partial_{3} v^{3}\right\|_{L^{2}}\left\|\Delta_{k}^{\mathrm{h}} \Delta_{\ell}^{\mathrm{v}} \partial_{3} v^{3}\right\|_{L^{2}}
\end{aligned}
$$

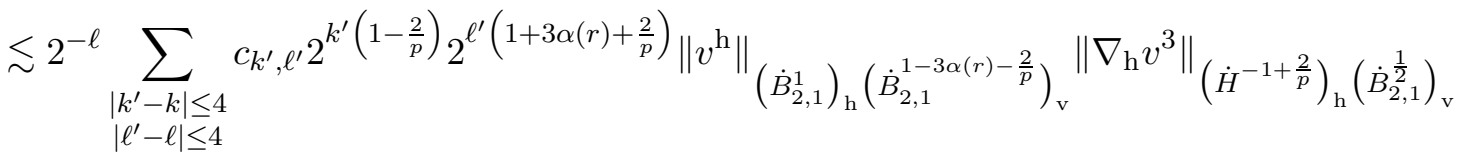

$$
\begin{aligned}
& \times c_{k, \ell} 2^{-k\left(1-\frac{2}{p}-6 \alpha(r)+2 \theta\right)} 2^{-\ell\left(\frac{2}{p}+3 \alpha(r)-2 \theta\right)}\left\|\partial_{3} v^{3}\right\|_{\dot{H}^{1-\frac{2}{p}-6 \alpha(r)+2 \theta, \frac{2}{p}+3 \alpha(r)-2 \theta}}
\end{aligned}
$$

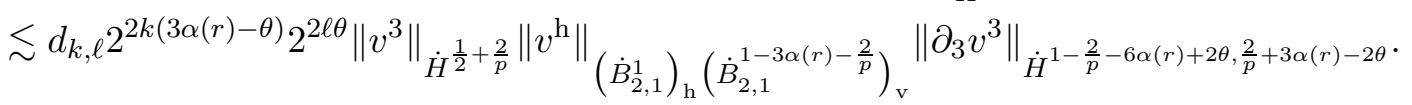

Therefore, by virtue of (5.8), we obtain

$$
\begin{aligned}
\left|I_{k, \ell}^{1}\right| \lesssim & d_{k, \ell} 2^{2 k(3 \alpha(r)-\theta)} 2^{2 \ell \theta}\left\|v^{3}\right\|_{\dot{H}^{\frac{1}{2}+\frac{2}{p}}}\left(\left\|\nabla_{\mathrm{h}} v^{\mathrm{h}}\right\|_{\dot{H}^{\frac{1}{2}-3 \alpha(r)+\theta, \frac{1}{2}-\frac{1}{p}-\theta}}^{2}\right. \\
& +\left\|\partial_{3} v^{3}\right\|_{\dot{H}^{\frac{1}{2}-3 \alpha(r)+\theta, \frac{1}{2}-\frac{1}{p}-\theta}}^{2}+\left\|v^{\mathrm{h}}\right\|_{\left.\left(\dot{B}_{2,1}^{1}\right)_{\mathrm{h}}\left(\dot{B}_{2,1}^{1-3 \alpha(r)-\frac{2}{p}}\right)_{\mathrm{v}}\left\|\nabla \partial_{3} v^{3}\right\|_{\mathcal{H}^{\theta, r}}\right) .} .
\end{aligned}
$$

The same argument gives the same estimate for $I_{k, \ell}^{2}$. We thus conclude that

$$
\left(\Delta_{k}^{\mathrm{h}} \Delta_{\ell}^{\mathrm{v}} T^{\mathrm{h}} T^{\mathrm{v}}\left(v^{\mathrm{h}}, \nabla_{\mathrm{h}} \partial_{3} v^{3}\right) \mid \Delta_{k}^{\mathrm{h}} \Delta_{\ell}^{\mathrm{v}} \partial_{3} v^{3}\right)_{L^{2}}
$$

also verifies the Estimate (5.13).

- The estimate of $\left(\Delta_{k}^{\mathrm{h}} \Delta_{\ell}^{\mathrm{v}} A \mid \Delta_{k}^{\mathrm{h}} \Delta_{\ell}^{\mathrm{v}}\right)_{L^{2}}$. 
We first get, by applying Lemma 3.3, that

$$
\begin{aligned}
& \left\|S_{k^{\prime}-1}^{\mathrm{h}} \Delta_{\ell^{\prime}}^{\mathrm{v}} v^{\mathrm{h}}\right\|_{L_{\mathrm{h}}^{\infty}\left(L_{\mathrm{v}}^{2}\right)} \lesssim 2^{-\ell^{\prime}\left(1-3 \alpha(r)-\frac{2}{p}\right)}\left\|v^{\mathrm{h}}\right\|_{\left(\dot{B}_{2,1}^{1}\right)_{\mathrm{h}}\left(\dot{B}_{2,1}^{1-3 \alpha(r)-\frac{2}{p}}\right)_{\mathrm{v}}}, \\
& \left\|\Delta_{k^{\prime}}^{\mathrm{h}} S_{\ell^{\prime}-1}^{\mathrm{v}} \nabla_{\mathrm{h}} \partial_{3} v^{3}\right\|_{L_{\mathrm{h}}^{2}\left(L_{\mathrm{v}}^{\infty}\right)} \lesssim c_{k^{\prime}, \ell^{\prime}} 2^{k^{\prime}\left(1-\frac{2}{p}\right)} 2^{\ell^{\prime}}\left\|v^{3}\right\|_{\left(\dot{H}^{\frac{2}{p}}\right)_{\mathrm{h}}\left(\dot{B}_{2,1}^{\frac{1}{2}}\right)_{\mathrm{v}} .}
\end{aligned}
$$

In view of (5.14), Lemma 3.3, and also considering the support to the Fourier transform to terms in $T^{\mathrm{h}} R^{\mathrm{v}}\left(v^{\mathrm{h}}, \nabla_{\mathrm{h}} \partial_{3} v^{3}\right)$, we write

$$
\begin{gathered}
\left\|\Delta_{k}^{\mathrm{h}} \Delta_{\ell}^{\mathrm{v}} T^{\mathrm{h}} R^{\mathrm{v}}\left(v^{\mathrm{h}}, \nabla_{\mathrm{h}} \partial_{3} v^{3}\right)\right\|_{L^{2}} \lesssim 2^{\ell / 2} \sum_{\substack{\left|k^{\prime}-k\right| \leq 4 \\
\ell^{\prime} \geq \ell-3}}\left\|S_{k^{\prime}-1}^{\mathrm{h}} \Delta_{\ell^{\prime}}^{\mathrm{v}} v^{\mathrm{h}}\right\|_{L_{\mathrm{h}}^{\infty}\left(L_{\mathrm{v}}^{2}\right)}\left\|\Delta_{k^{\prime}}^{\mathrm{h}} \widetilde{\Delta}_{\ell^{\prime}}^{\mathrm{v}} \nabla_{\mathrm{h}} \partial_{3} v^{3}\right\|_{L^{2}} \\
\lesssim 2^{\ell / 2} \sum_{\substack{\left|k^{\prime}-k\right| \leq 4 \\
\ell^{\prime} \geq \ell-3}} c_{k^{\prime}, \ell^{\prime}} 2^{2 k^{\prime}\left(\frac{1}{p}+3 \alpha(r)-\theta\right)} 2^{-\ell^{\prime}(1-2 \theta)}\left\|v^{\mathrm{h}}\right\|_{\left(\dot{B}_{2,1}^{1}\right)_{\mathrm{h}}\left(\dot{B}_{2,1}^{1-3 \alpha(r)-\frac{2}{p}}\right)_{\mathrm{v}}} \\
\times\left\|\partial_{3} v^{3}\right\|_{\dot{H}^{1-\frac{2}{p}-6 \alpha(r)+2 \theta, \frac{2}{p}+3 \alpha(r)-2 \theta}},
\end{gathered}
$$

which gives

$$
\begin{aligned}
& \left\|\Delta_{k}^{\mathrm{h}} \Delta_{\ell}^{\mathrm{v}} T^{\mathrm{h}} R^{\mathrm{v}}\left(v^{\mathrm{h}}, \nabla_{\mathrm{h}} \partial_{3} v^{3}\right)\right\|_{L^{2}} \lesssim c_{k, \ell} 2^{2 k\left(\frac{1}{p}+3 \alpha(r)-\theta\right)} 2^{-\ell\left(\frac{1}{2}-2 \theta\right)}
\end{aligned}
$$

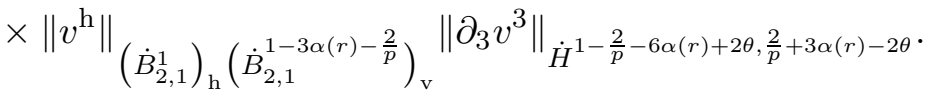

Therefore since

$$
\begin{aligned}
\left\|\Delta_{k}^{\mathrm{h}} \Delta_{\ell}^{\mathrm{v}} \partial_{3} v^{3}\right\|_{L^{2}} & \lesssim c_{k, \ell} 2^{-\frac{2 k}{p}} 2^{\frac{\ell}{2}}\left\|v^{3}\right\|_{\left(\dot{H}^{\frac{2}{p}}\right)_{\mathrm{h}}\left(\dot{B}_{2,1}^{\frac{1}{2}}\right)_{\mathrm{v}}} \\
& \lesssim c_{k, \ell} 2^{-\frac{2 k}{p}} 2^{\frac{\ell}{2}}\left\|v^{3}\right\|_{\dot{H}^{\frac{1}{2}+\frac{2}{p}}}
\end{aligned}
$$

we obtain

$$
\begin{aligned}
& \left|\left(\Delta_{k}^{\mathrm{h}} \Delta_{\ell}^{\mathrm{v}} T^{\mathrm{h}} R^{\mathrm{v}}\left(v^{\mathrm{h}}, \nabla_{\mathrm{h}} \partial_{3} v^{3}\right) \mid \Delta_{k}^{\mathrm{h}} \Delta_{\ell}^{\mathrm{v}} \partial_{3} v^{3}\right)_{L^{2}}\right| \lesssim d_{k, \ell} 2^{2 k(3 \alpha(r)-\theta)} 2^{2 \ell \theta}\left\|v^{3}\right\|_{\dot{H}^{\frac{1}{2}+\frac{2}{p}}}
\end{aligned}
$$

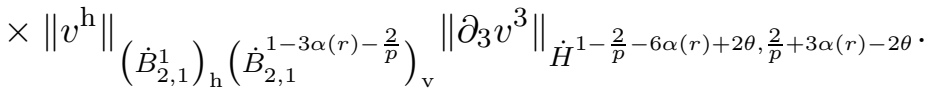

Along the same lines, we infer from (5.14) that

$$
\begin{aligned}
& \left\|\Delta_{k}^{\mathrm{h}} \Delta_{\ell}^{\mathrm{v}} T^{\mathrm{h}} \bar{T}^{\mathrm{v}}\left(v^{\mathrm{h}}, \nabla_{\mathrm{h}} \partial_{3} v^{3}\right)\right\|_{L^{2}} \lesssim \sum_{\substack{\left|k^{\prime}-k\right| \leq 4 \\
\left|\ell^{\prime}-\ell\right| \leq 4}}\left\|S_{k^{\prime}-1}^{\mathrm{h}} \Delta_{\ell^{\prime}}^{\mathrm{v}} v^{\mathrm{h}}\right\|_{L_{\mathrm{h}}^{\infty}\left(L_{\mathrm{v}}^{2}\right)}\left\|\Delta_{k^{\prime}}^{\mathrm{h}} S_{\ell^{\prime}-1}^{\mathrm{v}} \nabla_{\mathrm{h}} \partial_{3} v^{3}\right\|_{L_{\mathrm{h}}^{2}\left(L_{\mathrm{v}}^{\infty}\right)} \\
& \lesssim c_{k, \ell} 2^{k\left(1-\frac{2}{p}\right)} 2^{\ell\left(3 \alpha(r)+\frac{2}{p}\right)}
\end{aligned}
$$

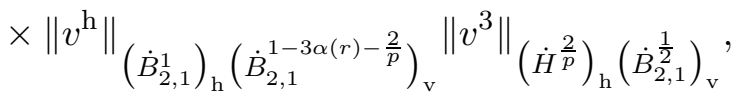

so that we obtain

$$
\begin{aligned}
& \left|\left(\Delta_{k}^{\mathrm{h}} \Delta_{\ell}^{\mathrm{v}} T^{\mathrm{h}} \bar{T}^{\mathrm{v}}\left(v^{\mathrm{h}}, \nabla_{\mathrm{h}} \partial_{3} v^{3}\right) \mid \Delta_{k}^{\mathrm{h}} \Delta_{\ell}^{\mathrm{v}} \partial_{3} v^{3}\right)_{L^{2}}\right| \lesssim d_{k, \ell} 2^{2 k(3 \alpha(r)-\theta)} 2^{2 \ell \theta}\left\|v^{3}\right\|_{\dot{H}^{\frac{1}{2}+\frac{2}{p}}}
\end{aligned}
$$

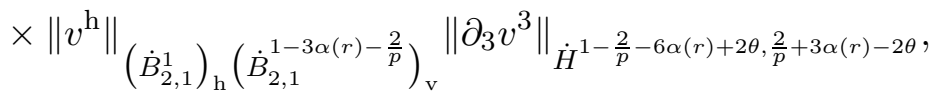


from which, (5.8) and (5.15), we deduce that

$$
\begin{aligned}
\left|\left(\Delta_{k}^{\mathrm{h}} \Delta_{\ell}^{\mathrm{v}} A \mid \Delta_{k}^{\mathrm{h}} \Delta_{\ell}^{\mathrm{v}} \partial_{3} v^{3}\right)_{L^{2}}\right| \lesssim & d_{k, \ell} 2^{2 k(3 \alpha(r)-\theta)} 2^{2 \ell \theta}\left\|v^{3}\right\|_{\dot{H}^{\frac{1}{2}+\frac{2}{p}}} \\
& \times\left\|v^{\mathrm{h}}\right\|_{\left(\dot{B}_{2,1}^{1}\right)_{\mathrm{h}}\left(\dot{B}_{2,1}^{1-3 \alpha(r)-\frac{2}{p}}\right)_{\mathrm{v}}\left\|\nabla \partial_{3} v^{3}\right\|_{\mathcal{H}^{\theta, r}} .}
\end{aligned}
$$

- The estimate of $\left(\Delta_{k}^{\mathrm{h}} \Delta_{\ell}^{\mathrm{v}} B \mid \Delta_{k}^{\mathrm{h}} \Delta_{\ell}^{\mathrm{v}}\right)_{L^{2}}$.

Again considering the support to the Fourier transform to terms in $R^{\mathrm{h}} R^{\mathrm{v}}\left(v^{\mathrm{h}}, \nabla_{\mathrm{h}} \partial_{3} v^{3}\right)$, we get, by applying Lemma 3.3, that

$$
\begin{aligned}
& \left\|\Delta_{k}^{\mathrm{h}} \Delta_{\ell}^{\mathrm{v}} R^{\mathrm{h}} R^{\mathrm{v}}\left(v^{\mathrm{h}}, \nabla_{\mathrm{h}} \partial_{3} v^{3}\right)\right\|_{L^{2}} \lesssim 2^{k} 2^{\ell / 2} \sum_{\substack{k^{\prime} \geq k-3 \\
\ell^{\prime} \geq \ell-3}}\left\|\Delta_{k^{\prime}}^{\mathrm{h}} \Delta_{\ell^{\prime}}^{\mathrm{v}} v^{\mathrm{h}}\right\|_{L^{2}}\left\|\widetilde{\Delta}_{k^{\prime}}^{\mathrm{h}} \widetilde{\Delta}_{\ell^{\prime}}^{\mathrm{v}} \nabla_{\mathrm{h}} \partial_{3} v^{3}\right\|_{L^{2}} \\
& \lesssim 2^{k} 2^{\ell / 2} \sum_{\substack{k^{\prime} \geq k-3 \\
\ell^{\prime} \geq \ell-3}} c_{k^{\prime}, \ell^{\prime}} 2^{-k^{\prime}(1-6 \alpha(r)+2 \theta)} 2^{-\ell^{\prime}\left(1-\frac{2}{p}-2 \theta\right)} \\
& \times\left\|\nabla_{\mathrm{h}} v^{\mathrm{h}}\right\|_{\dot{H}^{\frac{1}{2}-3 \alpha(r)+\theta, \frac{1}{2}-\frac{1}{p}-\theta}}\left\|\partial_{3} v^{3}\right\|_{\dot{H}^{\frac{1}{2}-3 \alpha(r)+\theta, \frac{1}{2}-\frac{1}{p}-\theta}} \\
& \lesssim c_{k, \ell} 2^{2 k(3 \alpha(r)-\theta)} 2^{-\ell\left(\frac{1}{2} n b s p ;-\frac{2}{p}-2 \theta\right)}\left\|\nabla_{\mathrm{h}} v^{\mathrm{h}}\right\|_{\dot{H}^{\frac{1}{2}-3 \alpha(r)+\theta, \frac{1}{2}-\frac{1}{p}-\theta}}\left\|\partial_{3} v^{3}\right\|_{\dot{H}^{\frac{1}{2}-3 \alpha(r)+\theta, \frac{1}{2}-\frac{1}{p}-\theta}},
\end{aligned}
$$

by using the fact that $3 \alpha(r)-\frac{1}{2}<0<\theta<\frac{1}{2}-\frac{2}{p}$.

Likewise, we have

$$
\begin{aligned}
& \left\|\Delta_{k}^{\mathrm{h}} \Delta_{\ell}^{\mathrm{v}} R^{\mathrm{h}} T^{\mathrm{v}}\left(v^{\mathrm{h}}, \nabla_{\mathrm{h}} \partial_{3} v^{3}\right)\right\|_{L^{2}} \lesssim 2^{k} \sum_{\substack{k^{\prime} \geq k-3 \\
\left|\ell^{\prime}-\ell\right| \leq 4}}\left\|\Delta_{k^{\prime}}^{\mathrm{h}} S_{\ell^{\prime}-1}^{\mathrm{v}} v^{\mathrm{h}}\right\|_{L_{\mathrm{h}}^{2}\left(L_{\mathrm{v}}^{\infty}\right)}\left\|\widetilde{\Delta}_{k^{\prime}}^{\mathrm{h}} \Delta_{\ell^{\prime}}^{\mathrm{v}} \nabla_{\mathrm{h}} \partial_{3} v^{3}\right\|_{L^{2}} \\
& \lesssim 2^{k} \sum_{\substack{k^{\prime} \geq k-3 \\
\left|\ell^{\prime}-\ell\right| \leq 4}} c_{k^{\prime}, \ell^{\prime}} 2^{-k^{\prime}(1-6 \alpha(r)+2 \theta)} 2^{-\ell^{\prime}\left(\frac{1}{2}-\frac{2}{p}-2 \theta\right)} \\
& \times\left\|\nabla_{\mathrm{h}} v^{\mathrm{h}}\right\|_{\dot{H}^{\frac{1}{2}-3 \alpha(r)+\theta, \frac{1}{2}-\frac{1}{p}-\theta}}\left\|\partial_{3} v^{3}\right\|_{\dot{H}^{\frac{1}{2}-3 \alpha(r)+\theta, \frac{1}{2}-\frac{1}{p}-\theta}} \\
& \lesssim c_{k, \ell} 2^{2 k(3 \alpha(r)-\theta)} 2^{-\ell\left(\frac{1}{2}-\frac{2}{p}-2 \theta\right)}\left\|\nabla_{\mathrm{h}} v^{\mathrm{h}}\right\|_{\dot{H}^{\frac{1}{2}-3 \alpha(r)+\theta, \frac{1}{2}-\frac{1}{p}-\theta}}\left\|\partial_{3} v^{3}\right\|_{\dot{H}^{\frac{1}{2}-3 \alpha(r)+\theta, \frac{1}{2}-\frac{1}{p}-\theta}} .
\end{aligned}
$$

It is easy to check that all the remaining terms in $B$ given by (5.12) share the same estimate. Therefore, we obtain

$$
\begin{aligned}
\left|\left(\Delta_{k}^{\mathrm{h}} \Delta_{\ell}^{\mathrm{v}} B \mid \Delta_{k}^{\mathrm{h}} \Delta_{\ell}^{\mathrm{v}}\right)_{L^{2}}\right| & \lesssim\left\|\Delta_{k}^{\mathrm{h}} \Delta_{\ell}^{\mathrm{v}} B\right\|_{L^{2}}\left\|\Delta_{k}^{\mathrm{h}} \Delta_{\ell}^{\mathrm{v}}\right\|_{L^{2}} \\
& \lesssim d_{k, \ell} 2^{2 k(3 \alpha(r)-\theta)} 2^{2 \ell \theta}\left\|\nabla_{\mathrm{h}} v^{\mathrm{h}}\right\|_{\dot{H}^{\frac{1}{2}-3 \alpha(r)+\theta, \frac{1}{2}-\frac{1}{p}-\theta}} \\
& \times\left\|\partial_{3} v^{3}\right\|_{\dot{H}^{\frac{1}{2}-3 \alpha(r)+\theta, \frac{1}{2}-\frac{1}{p}-\theta}}\left\|\partial_{3} v^{3}\right\|_{\dot{H}^{0,-\frac{1}{2}+\frac{2}{p}}}
\end{aligned}
$$

Inserting the Estimates (5.13), (5.16) and (5.17) in (5.12) leads to Lemma 5.2.

Thanks to Lemma 5.2, we get, by applying (2.4) and Proposition 3.1, that

$$
\begin{aligned}
& \left|\left(v^{\mathrm{h}} \cdot \nabla_{\mathrm{h}} \partial_{3} v^{3} \mid \partial_{3} v^{3}\right)_{\mathcal{H}^{\theta, r}}\right| \\
& \quad \lesssim\left\|v^{3}\right\|_{H^{\frac{1}{2}+\frac{2}{p}}}\left(\|\omega\|_{\dot{H}^{\frac{1}{2}-3 \alpha(r)+\theta, \frac{1}{2}-\frac{1}{p}-\theta}}^{2}+\left\|\partial_{3} v^{3}\right\|_{\dot{H}^{\frac{1}{2}-3 \alpha(r)+\theta, \frac{1}{2}-\frac{1}{p}-\theta}}^{2}\right. \\
& \left.\quad+\left(\left\|\omega_{\frac{r}{2}}\right\|_{L^{2}}^{2\left(\alpha(r)+\frac{1}{p}\right)}\left\|\nabla \omega_{\frac{r}{2}}\right\|_{L^{2}}^{1-\frac{2}{p}}+\left\|\partial_{3} v^{3}\right\|_{\mathcal{H}^{\theta, r}}^{\frac{2}{p}}\left\|\nabla \partial_{3} v^{3}\right\|_{\mathcal{H}^{\theta, r}}^{1-\frac{2}{p}}\right)\left\|\nabla \partial_{3} v^{3}\right\|_{\mathcal{H}^{\theta, r}}\right),
\end{aligned}
$$


from which, (??) and (5.6), we infer

$$
\begin{aligned}
\left|\left(v^{\mathrm{h}} \cdot \nabla_{\mathrm{h}} \partial_{3} v^{3} \mid \partial_{3} v^{3}\right)_{\mathcal{H}^{\theta, r}}\right| \lesssim\left\|v^{3}\right\|_{H^{\frac{1}{2}+\frac{2}{p}}}\left(\left\|\omega_{\frac{r}{2}}\right\|_{L^{2}}^{2\left(2 \alpha(r)+\frac{1}{p}\right)}\left\|\nabla \omega_{\frac{r}{2}}\right\|_{L^{2}}^{\frac{2}{p^{\prime}}}\right. \\
\left.+\left(\left\|\omega_{\frac{r}{2}}\right\|_{L^{2}}^{2\left(\alpha(r)+\frac{1}{p}\right)}\left\|\nabla \omega_{\frac{r}{2}}\right\|_{L^{2}}^{1-\frac{2}{p}}+\left\|\partial_{3} v^{3}\right\|_{\mathcal{H}^{\theta, r}}^{\frac{2}{p}}\left\|\nabla \partial_{3} v^{3}\right\|_{\mathcal{H}^{\theta, r}}^{1-\frac{2}{p}}\right)\left\|\nabla \partial_{3} v^{3}\right\|_{\mathcal{H}^{\theta, r}}\right) .
\end{aligned}
$$

To estimate $\left(v^{3} \partial_{3}^{2} v^{3} \mid \partial_{3} v^{3}\right)_{\mathcal{H}^{\theta, r}}$, we write, according to (5.3), that

$$
\left|(f \mid g)_{\mathcal{H}^{\theta, r}}\right| \leq\|f\|_{\dot{H}^{-1-3 \alpha(r)+\frac{2}{p}+\theta,-\theta}}\|g\|_{\dot{H}^{1-3 \alpha(r)-\frac{2}{p}+\theta,-\theta}} \text {. }
$$

As $\theta>3 \alpha(r)-\frac{2}{p}$, we get, by applying law of product of Lemma 3.7 and then Lemma 3.5, that

$$
\begin{aligned}
& \left|\left(v^{3} \partial_{3}^{2} v^{3} \mid \partial_{3} v^{3}\right)_{\mathcal{H}^{\theta, r}}\right| \leq\left\|v^{3} \partial_{3}^{2} v^{3}\right\|_{\dot{H}^{-1-3 \alpha(r)+\frac{2}{p}+\theta,-\theta}}\left\|\partial_{3} v^{3}\right\|_{\dot{H}^{1-3 \alpha(r)-\frac{2}{p}+\theta,-\theta}} \\
& \lesssim\left\|v^{3}\right\|_{\left(\dot{H}^{\frac{2}{p}}\right)_{\mathrm{h}}\left(B_{2,1}^{\frac{1}{2}}\right)_{\mathrm{v}}}\left\|\partial_{3}^{2} v^{3}\right\|_{\mathcal{H}^{\theta, r}}\left\|\partial_{3} v^{3}\right\|_{\dot{H}^{1-3 \alpha(r)-\frac{2}{p}+\theta,-\theta}} \\
& \lesssim\left\|v^{3}\right\|_{\dot{H}^{\frac{1}{2}+\frac{2}{p}}}\left\|\partial_{3}^{2} v^{3}\right\|_{\mathcal{H}^{\theta, r}}\left\|\partial_{3} v^{3}\right\|_{\dot{H}^{1-3 \alpha(r)-\frac{2}{p}+\theta,-\theta}} \text {. }
\end{aligned}
$$

This along with the interpolation inequality which claims that

$$
\begin{aligned}
\|a\|_{\dot{H}^{1-3 \alpha(r)-\frac{2}{p}+\theta,-\theta}}^{2} & =\int_{\mathbb{R}^{3}}\left|\xi_{\mathrm{h}}\right|^{2\left(1-\frac{2}{p}\right)}\left|\xi_{\mathrm{h}}\right|^{-6 \alpha(r)+2 \theta}\left|\xi_{3}\right|^{-2 \theta}|\widehat{a}(\xi)|^{2} d \xi \\
\leq & \left(\int_{\mathbb{R}^{3}}\left|\xi_{\mathrm{h}}\right|^{-6 \alpha(r)+2 \theta}\left|\xi_{3}\right|^{-2 \theta}|\widehat{a}(\xi)|^{2} d \xi\right)^{\frac{2}{p}} \\
& \times\left(\int_{\mathbb{R}^{3}}\left|\xi_{\mathrm{h}}\right|^{-6 \alpha(r)+2 \theta}\left|\xi_{3}\right|^{-2 \theta}\left|\xi_{\mathrm{h}}\right|^{2}|\widehat{a}(\xi)|^{2} d \xi\right)^{1-\frac{2}{p}} \\
\leq & \|a\|_{\mathcal{H}^{\theta, r}}^{4 / p}\left\|\nabla_{\mathrm{h}} a\right\|_{\mathcal{H}^{\theta, r}}^{2\left(1-\frac{2}{p}\right)}
\end{aligned}
$$

ensures

$$
\left|\left(v^{3} \partial_{3}^{2} v^{3} \mid \partial_{3} v^{3}\right)_{\mathcal{H}^{\theta, r}}\right| \lesssim\left\|v^{3}\right\|_{\dot{H}^{\frac{1}{2}+\frac{2}{p}}}\left\|\partial_{3} v^{3}\right\|_{\mathcal{H}^{\theta, r}}^{\frac{2}{p}}\left\|\nabla \partial_{3} v^{3}\right\|_{\mathcal{H}^{\theta, r}}^{\frac{2}{p^{\prime}}}
$$

Due to (5.18) and convexity inequality, we thus obtain

$$
\begin{aligned}
& \left|\left(Q_{3}(v, v) \mid \partial_{3} v^{3}\right)_{\mathcal{H}^{\theta, r}}\right| \leq \frac{1}{6}\left\|\nabla \partial_{3} v^{3}\right\|_{\mathcal{H}^{\theta, r}}^{2}+C\left\|v^{3}\right\|_{\dot{H}^{\frac{1}{2}+\frac{2}{p}}}^{p}\left\|\partial_{3} v^{3}\right\|_{\mathcal{H}^{\theta, r}}^{2} \\
& +C\left\|v^{3}\right\|_{\dot{H}^{\frac{1}{2}+\frac{2}{p}}}\left\|\omega_{\frac{r}{2}}\right\|_{L^{2}}^{2\left(2 \alpha(r)+\frac{1}{p}\right)}\left\|\nabla \omega_{\frac{r}{2}}\right\|_{L^{2}}^{\frac{2}{p^{2}}} \\
& +C\left\|v^{3}\right\|_{\dot{H}^{\frac{1}{2}+\frac{2}{p}}}^{2}\left\|\omega_{\frac{r}{2}}\right\|_{L^{2}}^{4\left(\alpha(r)+\frac{1}{p}\right)}\left\|\nabla \omega_{\frac{r}{2}}\right\|_{L^{2}}^{2\left(1-\frac{2}{p}\right)} .
\end{aligned}
$$

Now we are in a position to complete the proof of Proposition 2.2. 
Conclusion of the proof to Proposition 2.2. By resuming the Estimates (5.7), (5.10) and (5.19) into (5.1), we obtain

$$
\begin{aligned}
& \frac{d}{d t}\left\|\partial_{3} v^{3}(t)\right\|_{\mathcal{H}^{\theta, r}}^{2}+\left\|\nabla \partial_{3} v^{3}(t)\right\|_{\mathcal{H}^{\theta, r}}^{2} \\
& \leq C\left(\left\|v^{3}\right\|_{\dot{H}^{\frac{1}{2}+\frac{2}{p}}}\left\|\omega_{\frac{r}{2}}\right\|_{L^{2}}^{2\left(2 \alpha(r)+\frac{1}{p}\right)}\left\|\nabla \omega_{\frac{r}{2}}\right\|_{L^{2}}^{\frac{2}{p}}\right. \\
& \left.\quad+\left\|v^{3}\right\|_{\dot{H}^{\frac{1}{2}+\frac{2}{p}}}^{p}\left\|\partial_{3} v^{3}\right\|_{\mathcal{H}^{\theta, r}}^{2}+\left\|v^{3}\right\|_{\dot{H}^{\frac{1}{2}+\frac{2}{p}}}^{2}\left\|\omega_{\frac{r}{2}}\right\|_{L^{2}}^{4\left(\alpha(r)+\frac{1}{p}\right)}\left\|\nabla \omega_{\frac{r}{2}}\right\|_{L^{2}}^{2\left(1-\frac{2}{p}\right)}\right) .
\end{aligned}
$$

On the other hand, Inequality (2.7) cla ims that $\left\|\partial_{3} v_{0}^{3}\right\|_{\mathcal{H}^{\theta, r}} \lesssim\left\|v_{0}\right\|_{\dot{H}^{1-3 \alpha(r)}} \lesssim\left\|\Omega_{0}\right\|_{L^{r}}$. Thus Gronwall's inequality allows to conclude the proof of Proposition 2.2.

\section{Conclusion of the Proof of Theorem 2.1}

The first main step is the proof of the following proposition.

Proposition 6.1. Let us consider a solution $v$ of $(N S)$ given by Theorem 2.1. For any $p$ in $] 4, \frac{2 r}{2-r}[$ and $\theta$ in $] 3 \alpha(r)-\frac{2}{p}, \alpha(r)\left[\right.$, a constant $C$ exists such that, for any $t<T^{*}$, we have

$$
\begin{aligned}
\left\|\omega \frac{r}{2}(t)\right\|_{L^{2}}^{2(1+2 p \alpha(r))}+\left\|\nabla \omega \frac{r}{2}\right\|_{L_{t}^{2}\left(L^{2}\right)}^{2(1+2 p \alpha(r))} & \leq C\left\|\Omega_{0}\right\|_{L^{r}}^{r(1+2 p \alpha(r))} \mathcal{E}(t) \quad \text { and } \\
\left\|\partial_{3} v^{3}(t)\right\|_{\mathcal{H}^{\theta, r}}^{2}+\left\|\nabla \partial_{3} v^{3}\right\|_{L_{t}^{2}\left(\mathcal{H}^{\theta, r}\right)}^{2} & \leq\left\|\Omega_{0}\right\|_{L^{r}}^{2} \mathcal{E}(t) \quad \text { with } \\
\mathcal{E}(t) & \stackrel{\text { def }}{=} \exp \left(C \exp \left(C \int_{0}^{t}\left\|v^{3}\left(t^{\prime}\right)\right\|_{\dot{H}^{\frac{1}{2}+\frac{2}{p}}}^{p} d t^{\prime}\right) \beta i g g r\right) .
\end{aligned}
$$

Proof. The important point is the proof of the following estimate: for any $t$ in $\left[0, T^{\star}[\right.$, we have

$$
\begin{aligned}
\left\|\omega_{\frac{r}{2}}(t)\right\|_{L^{2}}^{2(1+2 p \alpha(r))}+ & \left\|\nabla \omega_{\frac{r}{2}}\right\|_{L_{t}^{2}\left(L^{2}\right)}^{2(1+2 p \alpha(r))} \\
& \leq C\left\|\Omega_{0}\right\|_{L^{r}}^{r(1+2 p \alpha(r))} \exp \left(C \exp \left(C \int_{0}^{t}\left\|v^{3}\left(t^{\prime}\right)\right\|_{\dot{H}^{\frac{1}{2}+\frac{2}{p}}}^{p} d t^{\prime}\right)\right) .
\end{aligned}
$$

In order to do it, let us introduce the notation

$$
e(T) \stackrel{\text { def }}{=} C \exp \left(C \int_{0}^{T}\left\|v^{3}(t)\right\|_{\dot{H}^{\frac{1}{2}+\frac{2}{p}}}^{p} d t\right) .
$$

where the constant $C$ may change from line to line. As $(a+b)^{\frac{r}{2}} \sim a^{\frac{r}{2}}+b^{\frac{r}{2}}$, Proposition 2.2 implies that

$$
\begin{aligned}
\left(\int_{0}^{t}\left\|\partial_{3}^{2} v^{3}\left(t^{\prime}\right)\right\|_{\mathcal{H}^{\theta, r}}^{2} d t^{\prime}\right)^{\frac{r}{2}} e(T) \lesssim e(T)\left(\left\|\Omega_{0}\right\|_{L^{r}}^{r}+V_{1}(t)+V_{2}(t)\right) \quad \text { with } \\
V_{1}(t) \stackrel{\text { def }}{=}\left(\int_{0}^{t}\left\|v^{3}\left(t^{\prime}\right)\right\|_{\dot{H}^{\frac{1}{2}+\frac{2}{p}}}\left\|\omega_{\frac{r}{2}}\left(t^{\prime}\right)\right\|_{L^{2}}^{2\left(2 \alpha(r)+\frac{1}{p}\right)}\left\|\nabla \omega_{\frac{r}{2}}\left(t^{\prime}\right)\right\|_{L^{2}}^{\frac{2}{p^{\prime}}} d t^{\prime}\right)^{\frac{r}{2}} \text { and } \\
V_{2}(t) \stackrel{\text { def }}{=}\left(\int_{0}^{t}\left\|v^{3}\left(t^{\prime}\right)\right\|_{\dot{H}^{\frac{1}{2}+\frac{2}{p}}}^{2}\left\|\omega_{\frac{r}{2}}\left(t^{\prime}\right)\right\|_{L^{2}}^{4\left(\alpha(r)+\frac{1}{p}\right)}\left\|\nabla \omega_{\frac{r}{2}}\left(t^{\prime}\right)\right\|_{L^{2}}^{2\left(1-\frac{2}{p}\right)} d t^{\prime}\right)^{\frac{r}{2}}
\end{aligned}
$$

Let us estimate the two terms $V_{j}(t), j=1,2$. Applying Hölder inequality gives

$$
V_{1}(t) \leq\left(\int_{0}^{t}\left\|v^{3}\left(t^{\prime}\right)\right\|_{\dot{H}^{\frac{1}{2}+\frac{2}{p}}}^{p}\left\|\omega_{\frac{r}{2}}\left(t^{\prime}\right)\right\|_{L^{2}}^{2(1+2 p \alpha(r))} d t^{\prime}\right)^{\frac{r}{2} \times \frac{1}{p}}\left(\int_{0}^{t}\left\|\nabla \omega_{\frac{r}{2}}\left(t^{\prime}\right)\right\|_{L^{2}}^{2} d t^{\prime}\right)^{\frac{r}{2}\left(1-\frac{1}{p}\right)} .
$$


As we have

$$
1-\frac{r}{2}\left(1-\frac{1}{p}\right)=r\left(\alpha(r)+\frac{1}{2 p}\right)=\frac{r(1+2 p \alpha(r))}{2 p}
$$

convexity inequality implies that, for any $t$ in $[0, T]$,

$$
\begin{aligned}
e(T) V_{1}(t) \leq \frac{r-1}{3 r^{2}} & \int_{0}^{t}\left\|\nabla \omega_{\frac{r}{2}}\left(t^{\prime}\right)\right\|_{L^{2}}^{2} d t^{\prime} \\
& +e(T)\left(\int_{0}^{t}\left\|v^{3}\left(t^{\prime}\right)\right\|_{\dot{H}^{\frac{1}{2}+\frac{2}{p}}}^{p}\left\|\omega_{\frac{r}{2}}\left(t^{\prime}\right)\right\|_{L^{2}}^{2(1+2 p \alpha(r))} d t^{\prime}\right)^{\frac{1}{1+2 p \alpha(r)}} .
\end{aligned}
$$

Now let us estimate the term $V_{2}(t)$. Applying Hölder inequality yields

$$
V_{2}(t) \leq\left(\int_{0}^{t}\left\|v^{3}\left(t^{\prime}\right)\right\|_{\dot{H}^{\frac{1}{2}+\frac{2}{p}}}^{p}\left\|\omega_{\frac{r}{2}}\left(t^{\prime}\right)\right\|_{L^{2}}^{2(1+p \alpha(r))} d t^{\prime}\right)^{\frac{r}{2} \times \frac{2}{p}}\left(\int_{0}^{t}\left\|\nabla \omega_{\frac{r}{2}}\left(t^{\prime}\right)\right\|_{L^{2}}^{2} d t^{\prime}\right)^{\frac{r}{2}\left(1-\frac{2}{p}\right)} .
$$

As we have

$$
1-\frac{r}{2}\left(1-\frac{2}{p}\right)=r\left(\alpha(r)+\frac{1}{p}\right)=\frac{r(1+p \alpha(r))}{p},
$$

convexity inequality implies that

$$
\begin{aligned}
e(T) V_{2}(t) \leq \frac{r-1}{3 r^{2}} & \int_{0}^{t}\left\|\nabla \omega_{\frac{r}{2}}\left(t^{\prime}\right)\right\|_{L^{2}}^{2} d t^{\prime} \\
& +e(T)\left(\int_{0}^{t}\left\|v^{3}\left(t^{\prime}\right)\right\|_{\dot{H}^{\frac{1}{2}+\frac{2}{p}}}^{p}\left\|\omega_{\frac{r}{2}}\left(t^{\prime}\right)\right\|_{L^{2}}^{2(1+p \alpha(r))} d t^{\prime}\right)^{\frac{1}{1+p \alpha(r)}} .
\end{aligned}
$$

Let us notice that the power of $\left\|\omega_{\frac{r}{2}}\right\|_{L^{2}}$ here is not the same as that in Inequality (6.24). Applying Hölder inequality with

$$
q=\frac{1+2 p \alpha(r)}{1+p \alpha(r)}
$$

and with the measure $\left\|v^{3}\left(t^{\prime}\right)\right\|_{\delta H^{\frac{1}{2}+\frac{2}{p}}}^{p} d t^{\prime}$ gives

$$
\begin{gathered}
\left(\int_{0}^{t}\left\|v^{3}\left(t^{\prime}\right)\right\|_{\dot{H}^{\frac{1}{2}+\frac{2}{p}}}^{p}\left\|\omega_{\frac{r}{2}}\left(t^{\prime}\right)\right\|_{L^{2}}^{2(1+p \alpha(r))} d t^{\prime}\right)^{\frac{1}{1+p \alpha(r)}} \leq\left(\int_{0}^{t}\left\|v^{3}\left(t^{\prime}\right)\right\|_{\dot{H}^{\frac{1}{2}+\frac{2}{p}}}^{p} d t^{\prime}\right)^{\left(1-\frac{1}{q}\right) \times \frac{1}{1+p \alpha(r)}} \\
\times\left(\int_{0}^{t}\left\|v^{3}\left(t^{\prime}\right)\right\|_{\dot{H}^{\frac{1}{2}+\frac{2}{p}}}^{p}\left\|\omega_{\frac{r}{2}}\left(t^{\prime}\right)\right\|_{L^{2}}^{2(1+2 p \alpha(r))} d t^{\prime}\right)^{\frac{1}{1+2 p \alpha(r)}} .
\end{gathered}
$$

By definition of $e(T)$, we have

$$
\left(\int_{0}^{t}\left\|v^{3}\left(t^{\prime}\right)\right\|_{\dot{H}^{\frac{1}{2}+\frac{2}{p}}}^{p} d t^{\prime}\right)^{\left(1-\frac{1}{q}\right) \times \frac{1}{1+p \alpha(r)}} e(T) \leq e(T) .
$$

Thus we deduce from (6.25) that

$$
e(T) V_{2}(t) \leq \frac{r-1}{3 r^{2}} \int_{0}^{t}\left\|\nabla \omega_{\frac{r}{2}}\left(t^{\prime}\right)\right\|_{L^{2}}^{2} d t^{\prime}+e(T)\left(\int_{0}^{t}\left\|v^{3}\left(t^{\prime}\right)\right\|_{\dot{H}^{\frac{1}{2}+\frac{2}{p}}}^{p}\left\|\omega_{\frac{r}{2}}\left(t^{\prime}\right)\right\|_{L^{2}}^{2(1+2 p \alpha(r))} d t^{\prime}\right)^{\frac{1}{1+2 p \alpha(r)}} .
$$


Inserting this inequality and (6.24) in (6.23) gives, for any $t$ in $[0, T]$,

$$
\begin{aligned}
&\left(\int_{0}^{t}\left\|\partial_{3}^{2} v^{3}\left(t^{\prime}\right)\right\|_{\mathcal{H}^{\theta, r}}^{2} d t^{\prime}\right)^{\frac{r}{2}} e(T) \leq \frac{2(r-1)}{3 r^{2}} \int_{0}^{t}\left\|\nabla \omega_{\frac{r}{2}}\left(t^{\prime}\right)\right\|_{L^{2}}^{2} d t^{\prime}+e(T)\left\|\Omega_{0}\right\|_{L^{r}}^{r} \\
&+e(T)\left(\int_{0}^{t}\left\|v^{3}\left(t^{\prime}\right)\right\|_{\dot{H}^{\frac{1}{2}+\frac{2}{p}}}^{p}\left\|\omega_{\frac{r}{2}}\left(t^{\prime}\right)\right\|_{L^{2}}^{2(1+2 p \alpha(r))} d t^{\prime}\right)^{\frac{1}{1+2 p \alpha(r)}} .
\end{aligned}
$$

Hence thanks to Proposition 2.1, we deduce that

$$
\begin{aligned}
& \frac{1}{r}\left\|\omega_{\frac{r}{2}}(t)\right\|_{L^{2}}^{2}+\frac{r-1}{3 r^{2}} \int_{0}^{t}\left\|\nabla \omega_{\frac{r}{2}}\left(t^{\prime}\right)\right\|_{L^{2}}^{2} d t^{\prime} \leq\left\|\Omega_{0}\right\|_{L^{r}}^{r} e(T) \\
& \quad+e(T)\left(\int_{0}^{t}\left\|v^{3}\left(t^{\prime}\right)\right\|_{\dot{H}^{\frac{1}{2}+\frac{2}{p}}}^{p}\left\|\omega_{\frac{r}{2}}\left(t^{\prime}\right)\right\|_{L^{2}}^{2(1+2 p \alpha(r))} d t^{\prime}\right)^{\frac{1}{1+2 p \alpha(r)}} .
\end{aligned}
$$

Taking the power $1+2 p \alpha(r)$ of this inequality and using that

$$
(a+b)^{1+2 p \alpha(r)} \sim a^{1+2 p \alpha(r)}+b^{1+2 p \alpha(r)},
$$

we obtain for any $t$ in $[0, T]$,

$$
\begin{aligned}
\left\|\omega_{\frac{r}{2}}(t)\right\|_{L^{2}}^{2(1+2 p \alpha(r))}+\left(\int_{0}^{t}\left\|\nabla \omega_{\frac{r}{2}}\left(t^{\prime}\right)\right\|_{L^{2}}^{2} d t^{\prime}\right)^{1+2 p \alpha(r)} \leq\left\|\Omega_{0}\right\|_{L^{r}}^{r(1+2 p \alpha(r))} e(T) \\
+e(T) \int_{0}^{t}\left\|v^{3}\left(t^{\prime}\right)\right\|_{\dot{H}^{\frac{1}{2}+\frac{2}{p}}}^{p}\left\|\omega_{\frac{r}{2}}\left(t^{\prime}\right)\right\|_{L^{2}}^{2(1+2 p \alpha(r))} d t^{\prime} .
\end{aligned}
$$

Then Gronwall lemma leads to Inequality (6.21). On the other hand, it follows from Proposition 2.2 that, for any $t<T^{*}$,

$$
\begin{aligned}
&\left\|\partial_{3} v^{3}(t)\right\|_{\mathcal{H}^{\theta, r}}^{2}+\int_{0}^{t}\left\|\nabla \partial_{3} v^{3}\left(t^{\prime}\right)\right\|_{\mathcal{H}^{\theta, r}}^{2} d t^{\prime} \\
& \leq e(t)\left(\left\|\Omega_{0}\right\|_{L^{r}}^{2}+\left\|v^{3}\right\|_{L_{t}^{p}\left(\dot{H}^{\left.\frac{1}{2}+\frac{2}{p}\right)}\right.}\left\|\omega_{\frac{r}{2}}\right\|_{L_{t}^{\infty}\left(L^{2}\right)}^{2\left(2 \alpha(r)+\frac{1}{p}\right)}\left\|\nabla \omega_{\frac{r}{2}}\right\|_{L_{t}^{2}\left(L^{2}\right)}^{\frac{2}{p^{r}}}\right. \\
&\left.\quad+\left\|v^{3}\right\|_{L_{t}^{p}\left(\dot{H}^{\left.\frac{1}{2}+\frac{2}{p}\right)}\right.}^{2}\left\|\omega_{\frac{r}{2}}\right\|_{L_{t}^{\infty}\left(L^{2}\right)}^{\left.4\left(\alpha(r)+\frac{1}{p}\right)\right)}\left\|\nabla \omega_{\frac{r}{2}}\right\|_{L_{t}^{2}\left(L^{2}\right)}^{2\left(1-\frac{2}{p}\right)}\right) .
\end{aligned}
$$

Inserting the Estimate (6.21) in the above inequality concludes the proof of Proposition 6.1 .

Thus, if we assume that

$$
\int_{0}^{T^{\star}}\left\|v^{3}(t)\right\|_{\dot{H}^{\frac{1}{2}+\frac{2}{p}}}^{p} d t<\infty
$$

we know that the quantities

$$
\|\omega\|_{L^{\infty}\left(\left[0, T^{\star}\left[; L^{r}\right)\right.\right.}, \quad \int_{0}^{T^{\star}}\left\|\nabla \omega_{\frac{r}{2}}(t)\right\|_{L^{2}}^{2} d t, \quad \text { and } \quad \int_{0}^{T^{\star}}\left\|\partial_{3}^{2} v^{3}(t)\right\|_{\mathcal{H}^{\theta, r}}^{2} d t
$$

are finite. We want to prove that it prevents this solution from blowing up. Let us recall the following theorem of anisotropic condition for blow up. 
Theorem 6.1 (Theorem 2.1 of [5]). Let $v$ be a solution of $(N S)$ in the space $C\left(\left[0, T^{\star}\left[; \dot{H}^{\frac{1}{2}}\right) \cap\right.\right.$ $L_{\text {loc }}^{2}\left(\left[0, T^{\star}\left[; H^{3 / 2}\right)\right.\right.$. If $T^{\star}$ is the maximal time of existence and $T^{*}<\infty$, then for any $\left(p_{k, \ell}\right)$ in $] 1, \infty\left[{ }^{9}\right.$, one has

$$
\sum_{1 \leq k, \ell \leq 3} \int_{0}^{T^{\star}}\left\|\partial_{\ell} v^{k}(t)\right\|_{\mathcal{B}_{p_{k, \ell}}}^{p_{k, \ell}} d t=\infty
$$

where $\mathcal{B}_{p} \stackrel{\text { def }}{=} \dot{B}_{\infty, \infty}^{-2+\frac{2}{p}}$.

Now let us present the proof of Theorem 2.1.

Proof of Theorem 2.1. We first deduce from Lemma 3.3 that

$$
\max _{1 \leq \ell \leq 3}\left\|\partial_{\ell} v^{3}\right\|_{\mathcal{B}_{p}} \lesssim \sup _{j \in \mathbb{Z}} 2^{j\left(-1+\frac{2}{p}\right)}\left\|\Delta_{j} v^{3}\right\|_{L^{\infty}} \lesssim \sup _{j \in \mathbb{Z}} 2^{j\left(\frac{1}{2}+\frac{2}{p}\right)}\left\|\Delta_{j} v^{3}\right\|_{L^{2}} \lesssim\left\|v^{3}\right\|_{\dot{H}^{\frac{1}{2}+\frac{2}{p}}},
$$

which ensures that

$$
\max _{1 \leq \ell \leq 3} \int_{0}^{T^{\star}}\left\|\partial_{\ell} v^{3}(t)\right\|_{\mathcal{B}_{p}}^{p} d t \lesssim \int_{0}^{T^{\star}}\left\|v^{3}(t)\right\|_{\dot{H}^{\frac{1}{2}+\frac{2}{p}}}^{p} d t<\infty .
$$

As we have

$$
\left\|\partial_{\mathrm{h}}^{2} \Delta_{\mathrm{h}}^{-1} \partial_{3} v^{3}(t)\right\|_{\mathcal{B}_{p}} \lesssim\left\|\partial_{\mathrm{h}}^{2} \Delta_{\mathrm{h}}^{-1} \partial_{3} v^{3}(t)\right\|_{\dot{H}^{-\frac{1}{2}+\frac{2}{p}}},
$$

so that for $v_{\mathrm{div}}^{\mathrm{h}}=-\nabla_{\mathrm{h}} \Delta_{\mathrm{h}}^{-1} \partial_{3} v^{3}$, there holds

$$
\int_{0}^{T^{*}}\left\|\nabla_{\mathrm{h}} v_{\operatorname{div}}^{\mathrm{h}}(t)\right\|_{\mathcal{B}_{p}}^{p} d t \lesssim \int_{0}^{T^{*}}\left\|v^{3}(t)\right\|_{\dot{H}^{\frac{1}{2}+\frac{2}{p}}}^{p} d t<\infty .
$$

The other components of the matrix $\nabla v$ can been estimated with norm which are not of scaling 0 , namely norms related to $L^{r}$ regularity of the horizontal vorticity $\omega$. To proceed further, we get, by using Lemma 3.3, that

$$
\begin{aligned}
\left\|\Delta_{j} a\right\|_{L^{\infty}} & \lesssim \sum_{\substack{k \leq j+1 \\
\ell \leq j+1}} 2^{k} 2^{\frac{\ell}{2}}\left\|\Delta_{k}^{\mathrm{h}} \Delta_{\ell}^{\mathrm{v}} a\right\|_{L^{2}} \\
& \lesssim\|a\|_{\dot{H}^{1-3 \alpha(r)+\theta,-\theta}} \sum_{\substack{k \leq j+1 \\
\ell \leq j+1}} 2^{k(3 \alpha(r)-\theta)} 2^{\ell\left(\frac{1}{2}+\theta\right)} \\
& \lesssim 2^{j\left(\frac{1}{2}+3 \alpha(r)\right)}\|a\|_{\dot{H}^{1-3 \alpha(r)+\theta,-\theta}},
\end{aligned}
$$

because $-\frac{1}{2}-3 \alpha(r)=-2+\frac{3}{r^{\prime}}$, this leads to

$$
\|a\|_{\mathcal{B}_{\frac{2 r^{\prime}}{3}}} \lesssim\|a\|_{\dot{H}^{1-3 \alpha(r)+\theta,-\theta}} .
$$

Let us define $q(r) \stackrel{\text { def }}{=} \frac{2 r^{\prime}}{3}$. As $r$ belongs to $] 3 / 2,2[, q(r)$ is in $] 4 / 3,2[$ and thus is less than 2 . Observing that

$$
\left\|\partial_{3} v_{\operatorname{div}}^{\mathrm{h}}\right\|_{\dot{H}^{1-3 \alpha(r)+\theta,-\theta}}=\left\|\nabla_{\mathrm{h}} \Delta_{\mathrm{h}}^{-1} \partial_{3}^{2} v^{3}\right\|_{\dot{H}^{1-3 \alpha(r)+\theta,-\theta}} \lesssim\left\|\partial_{3}^{2} v^{3}\right\|_{\mathcal{H}^{\theta, r}},
$$

then applying Inequality (6.30) and Hölder inequality, we deduce that

$$
\int_{0}^{T^{*}}\left\|\partial_{3} v_{\operatorname{div}}^{\mathrm{h}}(t)\right\|_{\mathcal{B}_{q(r)}}^{q(r)} d t \lesssim T^{\star}\left(1-\frac{q(r)}{2}\right)\left(\int_{0}^{T^{*}}\left\|\partial_{3}^{2} v^{3}(t)\right\|_{\mathcal{H}^{\theta, r}}^{2} d t\right)^{\frac{q(r)}{2}}<\infty
$$


Let us admit for a while that

$$
\left\|\nabla v_{\mathrm{curl}}^{\mathrm{h}}(t)\right\|_{\mathcal{B}_{q(r)}} \lesssim\|\nabla \omega(t)\|_{L^{r}}
$$

Lemma 3.1 implies that

$$
\|\nabla \omega(t)\|_{L^{r}} \lesssim\left\|\omega_{\frac{r}{2}}\right\|_{L^{\infty}\left(\left[0, T^{\star} ; L^{2}\right)\right.}^{\frac{2}{r}-1}\left\|\nabla \omega_{\frac{r}{2}}(t)\right\|_{L^{2}} .
$$

Then Hölder inequality implies that

$$
\int_{0}^{T^{*}}\left\|\nabla v_{\text {curl }}^{\mathrm{h}}(t)\right\|_{\mathcal{B}_{q(r)}}^{q(r)} d t \lesssim T^{\star}\left(1-\frac{q(r)}{2}\right)\left\|\omega_{\frac{r}{2}}\right\|_{L^{\infty}\left(\left[0, T^{\star}\left[; L^{2}\right)\right.\right.}^{\frac{2}{r}-1}\left(\int_{0}^{T^{*}}\left\|\nabla \omega_{\frac{r}{2}}(t)\right\|_{L^{2}}^{2} d t\right)^{\frac{q(r)}{2}}<\infty .
$$

Together with Inequalities (6.28), (6.29) and (6.31), this concludes the proof of Theorem 2.1 provided we prove the Estimate (6.32).

Let us start with the term $\nabla_{\mathrm{h}} v_{\text {curl }}^{\mathrm{h}}$. Dual Sobolev embedding implies that

$$
\|\omega\|_{\dot{H}^{1-3 \alpha(r)}} \lesssim\|\nabla \omega\|_{\dot{H}^{-3 \alpha(r)}} \lesssim\|\nabla \omega\|_{L^{r}}
$$

As $\nabla_{\mathrm{h}} v_{\text {curl }}^{\mathrm{h}}=\partial_{\mathrm{h}}^{2} \Delta_{\mathrm{h}}^{-1} \omega$, we get, by using Lemma 3.5 and (6.30), that

$$
\left\|\nabla_{\mathrm{h}} v_{\mathrm{curl}}^{\mathrm{h}}\right\|_{\mathcal{B}_{q(r)}} \lesssim\left\|\partial_{\mathrm{h}}^{2} \Delta_{\mathrm{h}}^{-1} \omega\right\|_{\dot{H}^{1-3 \alpha(r)}} \lesssim\|\nabla \omega\|_{L^{r}}
$$

The term $\partial_{3} v_{\text {curl }}^{\mathrm{h}}$ is treated as follows. Let us write that

$$
\Delta_{j} \partial_{3} v_{\mathrm{curl}}^{\mathrm{h}}=\sum_{\substack{k \leq j+1 \\ \ell \leq j+1}} \Delta_{j} \Delta_{k}^{\mathrm{h}} \Delta_{\ell}^{\mathrm{v}} \partial_{3} \nabla_{\mathrm{h}}^{\perp} \Delta_{\mathrm{h}}^{-1} \omega
$$

Using Lemma 3.3, we can wri te

$$
\begin{aligned}
2^{-j\left(\frac{3}{r}-1\right)}\left\|\Delta_{j} \partial_{3} v_{\mathrm{curl}}^{\mathrm{h}}\right\|_{L^{\infty}} & \lesssim 2^{-j\left(\frac{3}{r}-1\right)} \sum_{\substack{k \leq j+1 \\
\ell \leq j+1}}\left\|\Delta_{j} \Delta_{k}^{\mathrm{h}} \Delta_{\ell}^{\mathrm{v}} \partial_{3} \nabla_{\mathrm{h}}^{\perp} \Delta_{\mathrm{h}}^{-1} \omega\right\|_{L^{\infty}} \\
& \lesssim\left\|\partial_{3} \omega\right\|_{L^{r}} 2^{-j\left(\frac{3}{r}-1\right)} \sum_{\substack{k \leq j+1 \\
\ell \leq j+1}} 2^{k\left(\frac{2}{r}-1\right)} 2^{\frac{\ell}{r}} \\
& \lesssim\left\|\partial_{3} \omega\right\|_{L^{r}}
\end{aligned}
$$

This concludes the proof of (6.32) and hence also the proof of Theorem 2.1.

Acknowledgments. Part of this work was done when J.-Y. Chemin was visiting Morningside Center of the Academy of Mathematics and Systems Sciences, CAS. We appreciate the hospitality and the financial support from MCM and National Center for Mathematics and Interdisciplinary Sciences. P. Zhang is partially supported by NSF of China under Grant 11371347, the fellowship from Chinese Academy of Sciences and innovation grant from National Center for Mathematics and Interdisciplinary Sciences. Z. Zhang is partially supported by NSF of China under Grant 11371037 and 11425103, Program for New Century Excellent Talents in University and Fok Ying Tung Education Foundation. 


\section{REFERENCES}

[1] H. Bahouri, J. Y. Chemin and R. Danchin, Fourier analysis and nonlinear partial differential equations, Grundlehren der mathematischen Wissenschaften 343, Springer-Verlag Berlin Heidelberg, 2011.

[2] J.-M. Bony, Calcul symbolique et propagation des singularités pour les équations aux dérivées partielles non linéaires, Annales de l'École Normale Supérieure, 14, 1981, pages 209-246.

[3] J.-Y. Chemin, B. Desjardins, I. Gallagher and E. Grenier, Fluids with anisotropic viscosity, Modélisation Mathématique et Analyse Numérique, 34, 2000, pages 315-335.

[4] J.-Y. Chemin and P. Zhang, On the global wellposedness to the 3-D incompressible anisotropic NavierStokes equations, Communications in Mathematical Physics, 272, 2007, pages 529-566.

[5] J.-Y. Chemin and P. Zhang, On the critical one component regularity for 3-D Navier-Stokes system, arXiv:1310.6442[math.AP], accepted by Annales de l'École Normale Supérieure on 2014.

[6] L. Escauriaza, G. Seregin and V. Šverák, $L^{3, \infty}$-solutions of Navier-Stokes equations and backward uniqueness, (Russian) Uspekhi Mat. Nauk, 58, 2003, no. 2(350), pages 3-44; translation in Russian Math. Surveys, 58, 2003, pages 211-250.

[7] H. Fujita and T. Kato, On the Navier-Stokes initial value problem I, Archive for Rational Mechanic Analysis, 16, 1964, pages 269-315.

[8] M. Paicu, Équation anisotrope de Navier-Stokes dans des espaces critiques, Revista Matemática Iberoamericana, 21, 2005, pages 179-235.

(J.-Y. Chemin) Laboratoire J.-L. Lions, UMR 7598, Université Pierre et Marie Curie, 75230 Paris Cedex 05, FRANCE

E-mail address: chemin@ann.jussieu.fr

(P. Zhang) Academy of Mathematics \& Systems Science and Hua Loo-Keng Key Laboratory of Mathematics, The Chinese Academy of Sciences, Beijing 100190, CHinA

E-mail address: zp@amss.ac.cn

(Z. ZHAng) School of Mathematical Science, Peking University, Beijing 100871, P. R. CHinA E-mail address: zfzhang@math.pku.edu.cn 